\title{
Estudios de conectividad del paisaje en América Latina: retos de investigación
}

\section{Landscape connectivity studies in Latin America: research challenges}

\author{
Edgar G. Leijal y Manuel E. Mendoza ${ }^{*}$
}

\begin{abstract}
1 Universidad Nacional Autónoma de México. Centro de Investigaciones en Geografía Ambiental. Morelia, Michoacán, México. eleija(a)ciga.unam.mx
\end{abstract}

\author{
* Autor de correspondencia. \\ mmendoza@ciga.unam.mx
}

\section{RESUMEN}

Los estudios enfocados a la conectividad del paisaje son actualmente clave para la planificación de la conservación de los ecosistemas; sin embargo, únicamente un número limitado de estudios proporcionan datos empíricos sobre los beneficios de la conectividad en la conservación de los ecosistemas terrestres. En este estudio, se analizaron las publicaciones sobre estudios de conectividad particularmente para cuatro tópicos en América Latina de 2000 a 2018, para brindar una visión del estado actual, retos y avances del conocimiento. La búsqueda se realizó en diferentes bases de datos, de las cuales se seleccionaron 217 trabajos. Todas las referencias seleccionadas fueron revisadas y clasificadas considerando: a) año de publicación, b) tipo de investigación, c) país de América Latina donde se realizó, d) área de estudio, e) tipo de ecosistema y f) objetivos de la investigación. Los resultados muestran que la mayor parte de los estudios de conectividad se realizan considerando los tópicos: a) de planificación para la restauración, b) modelado y planificación de la cobertura vegetal/uso del suelo y c) servicios ecosistémicos. Sin embargo, los trabajos publicados sobre conectividad y cambio climático han sido escasos. También fue posible identificar publicaciones que abordaron dos tópicos simultáneamente: a) estudios de modelado y planificación de la cobertura vegetal/uso del suelo con estudios de la planificación para la restauración, b) estudios en servicios ecosistémicos con cambio climático y c) planificación para la restauración con cambio climático. Sin embargo, existe la necesidad de incrementar sustancialmente las investigaciones en los temas de conectividad en América Latina.

PALABRAS CLAVE: buscadores de internet, flujos ecológicos, hábitat, Latinoamérica, revisión bibliográfica.

\section{ABSTRACT}

Currently, landscape connectivity studies are key in spatial planning for ecosystems conservation. Nevertheless, there are only a few studies that give empiric data about the positive effects of connectivity on terrestrial conservation ecosystems. In this piece of the research, we present a review of the literature of connectivity on four recently identified research topics carried out from 2000 to 2018 in Latin America. We identify the current stage, recent advance and challenges about connectivity knowledge. The scientific review used several databases on the web, from wich we selected 217 articles. The selected publications were included in a database considering: a) publication year, b) research type, c) country studied, d) ecosystem studied and f) and research aims. Our results depicted that connectivity studies focus on the topics: a) in restoration planning, b) land cover/land use change modeling and planning and c) ecosystem services. Nevertheless, the publications about connectivity and climate change have been limited. We also identified some publications tackling two or three topics simultaneously: a) land cover/land use change modeling and planning with restoration planning, b) ecosystem services with climate change, and c) restoration planning with climate change. However, there is a necessity to increase the connectivity research on Latin America.

KEYWORDS: internet search engines, ecologic flows, habitat, Latin America, literature review. 


\section{INTRODUCCIÓN}

La deforestación en América Latina, por procesos de cambio en el uso del terreno, derivado de múltiples factores ambientales, sociales y económicos, se ha convertido en tema de interés para la conservación de los ecosistemas (Lambin et al., 2001; Geist y Lambin, 2002; Haila, 2002; Fazey et al., 2005; Morera et al., 2007). En esta región, la tasa de deforestación anual es de $2.5 \%$, siendo el continente con mayor pérdida anual de área forestal para el periodo 19902000 con 25\% (Kim et al., 2015 Eva et al., 2012).

La expansión de la frontera agrícola y el incremento exponencial de la población e industrialización ha generado una mayor deforestación y fragmentación de los ecosistemas, ocasionando efectos de borde y aislamiento entre fragmentos (Sodhi et al., 2004; Lindenmayer y Fisher, 2006; Newbold et al., 2015; Leija y Pavón, 2017). Su impacto repercute en la modificación de las condiciones ambientales y en el funcionamiento de los ecosistemas, como los ciclos hidrológicos, el microclima, las propiedades del suelo, entre otros (Forman, 1994). La fragmentación también se produce a microescala por actividades antrópicas, como la tala ilegal o los incendios, ocasionando la pérdida de la biodiversidad (Laurance et al., 2011; Mendoza et al., 2011; Leija et al., 2016; Leija et al., 2020).

Asimismo, este fenómeno irrumpe y modifica la dinámica de las poblaciones (abundancia y funcionalidad de los organismos) y reduce su dispersión e intercambio de los procesos de inmigración, causando la extinción de especies (Mech y Hallet, 2001; Múgica et al., 2002; Cushman, 2006; Leidner, 2011; Correa-Ayram et al., 2016, 2017). Este impacto negativo afecta a diversos grupos taxonómicos, como las aves, los mamíferos, los reptiles, los anfibios, los invertebrados y las plantas (Gibbons et al., 2000; Hobbs y Yates, 2003; Stuart et al., 2004). Sin embargo, el impacto depende de los patrones de fragmentación de los hábitats, en la cual la abundancia de ciertas especies está relacionada con el tamaño y el grado de aislamiento de los parches de vegetación. Esto sin considerar el papel que juegan el efecto de los ecotonos, los cuales pueden perturbar o favorecer a otras especies (Lynch, 1992; Stouffer y Bierregaard, 1995).
Evitar la fragmentación de los hábitats y mantener la conectividad son prioridades de conservación para proteger la biodiversidad de los ecosistemas (Vos et al., 2008; Lindemayer et al., 2012; Correa-Ayram et al., 2014).

El cambio climático también juega un papel medular en los impactos negativos y adversos en la distribución de las especies terrestres (Pound y Salzmann, 2017; Junhu Su et al., 2018), vislumbrando la vulnerabilidad de los ecosistemas con los patrones climáticos (Etterson y Mazer, 2016; Wikelski y Tertiski, 2016). A través de disturbios directos e indirectos como los cambios fenológicos, la red alimentaria, la propagación de enfermedades y la extinción de especies (Wu et al., 2016). Sin embargo, algunas poblaciones podrán adaptarse al incremento de la temperatura terrestre, pero dependerá de la capacidad de resiliencia, resistencia, dispersión y colonización de otros hábitats geográficos por parte de las especies (Hoffman y Sgro, 2011; Mora et al., 2013).

La conectividad es clave en la conservación, particularmente en paisajes fragmentados. La conectividad del paisaje permite el intercambio y conexiones de flujos ecológicos, y cuenta con una función potencial para la evaluación entre hábitats (Taylor et al., 1993; Tischendorf y Fahrig, 2000; Belisle, 2005; Quesada et al., 2009). Su funcionalidad dependerá de las estructuras fisiográficas y espaciales del paisaje como en la permeabilidad del mosaico, la presencia de corredores y los puntos de paso o estriberones (Quesada et al., 2009; Smith et al., 2011); características esenciales para el movimiento, dispersión y comportamiento de las especies (Mech y Hallett, 2001; Gilbert-Norton et al., 2010).

Comprender y analizar los estudios orientados a la conectividad del paisaje permitiría crear programas y acciones orientados a la conservación de los ecosistemas a un menor costo, dado que la conectividad permite preservar las especies durante periodos prolongados y coadyuvaría al flujo e intercambio de especies entre hábitats dentro de un paisaje fragmentado. La conectividad es esencial y fundamental para el desarrollo de poblaciones viables de cualquier especie que requieran espacios de dispersión amplios o grupos de especies de tamaño más 
grandes (Hatfield et al., 2018; Mony et al., 2018). Por ello, es importante realizar un trabajo que revise el estado del arte, las tendencias y características de investigación sobre conectividad del paisaje para orientar esfuerzos en la conservación de los ecosistemas.

Mientras que la conectividad del paisaje (sensu stricto) tiene como objetivo el mantenimiento y funcionalidad de los ecosistemas en proyectos específicos, su definición aborda dos conceptos fundamentales como la conectividad estructural, la cual corresponde a la relación espacial entre elementos conspicuos del paisaje (parches de bosques), misma que es independiente de las características ecológicas de una especie (Taylor et al., 2006). Por otra parte, la conectividad funcional se refiere al movimiento de especies entre parches (Taylor et al., 2006); sin embargo, su aplicación se concentra principalmente en estudios elaborados en ecosistemas terrestres, particularmente en bosques tropicales y templados, y en menor medida para estudios fluviales y marinos (Correa-Ayram et al., 2016). Además de que prevalecen los estudios de conectividad en vertebrados y con amplio rango de dispersión, dejando de lado las especies menos visibles, pero con mayor importancia para entender la funcionalidad de los ecosistemas (Correa-Ayram et al., 2016).

\section{OBJETIVOS}

El objetivo principal de este trabajo fue analizar, a través de una revisión bibliográfica de publicaciones realizadas para el periodo 2000-2018, el desarrollo y evolución de las investigaciones sobre estudios de conectividad del paisaje en América Latina, particularmente para cuatro tópicos: a) conectividad y planificación de la restauración (CPR), b) modelado y planificación de conectividad de la cobertura vegetal/uso del suelo (MPCCUS), c) conectividad en servicios ecosistémicos (CSE) y d) conectividad y cambio climático (CC).

\section{MATERIALES Y MÉTODOS}

Se realizó una búsqueda exhaustiva de publicaciones científicas sobre conectividad del paisaje en América Latina de 2000 a 2018; particularmente en trabajos publicados para los cuatro tópicos identificados por Correa-Ayram et al. (2016). En la revisión se incluyeron publicaciones (artículos, libros y capítulos de libro). Se revisaron bases de datos en Scopus, Redalyc, EBSCO, Web of Science y del Sistema de Clasificación de Revistas Mexicanas de Ciencia y Tecnología Conacyt del Consejo Nacional de Ciencia y Tecnología (Conacyt). En todas las bases de datos, se emplearon las siguientes palabras clave: "conectividad", "hábitat", "fragmentación”, "paisaje”, "conectividad funcional", "ecosistema", "biodiversidad", “cambio climático", "medio ambiente", "conservación”, "cambio uso del suelo" y "modelado". Asimismo, se seleccionaron las siguientes áreas de investigación: biología, geografía, conservación de los recursos naturales, multidisciplinario, ciencias ambientales, agricultura y ciencias biológicas. Además, se realizó un filtro para limitar la búsqueda de información solo a países de América Latina; incluyendo únicamente estudios prácticos con aplicaciones directas de conectividad del paisaje en los cuatro tópicos anteriormente mencionados. Se descartaron referencias de publicaciones realizadas en ecosistemas acuáticos, debido a que no era el objeto de estudio en este trabajo. Solamente se consideraron trabajos realizados en ecosistemas terrestres sobre los cuatro tópicos en cuestión.

Posterior a la búsqueda de la literatura, el total de los trabajos fueron revisados y caracterizados. Se elaboró una base de datos conteniendo los siguientes campos: a) año de publicación, b) tipo de investigación, c) país de América Latina donde se realizó, d) área de estudio, e) tipo de ecosistema y f) objetivos de la investigación. La búsqueda permitió identificar una literatura amplia y diversa, lo que implicó realizar un filtro riguroso para seleccionar un subconjunto de artículos relacionados con los cuatro tópicos. Por ello, las palabras clave de búsqueda y la especificación de los estudios aplicados a la conectividad, dieron como resultado una evaluación de artículos que tienen un vínculo explícito entre los cuatro tópicos.

Sin embargo, pudo haber un sesgo al momento de realizar el filtro de búsqueda, de acuerdo con los criterios de selección, por lo que pudieron haber sido descartados algunos trabajos relacionados con estudios en conectividad. 
Para cada referencia se identificaron los puntos relevantes del trabajo, las preguntas de investigación consideradas y sus objetivos con base en los cuatro tópicos anteriormente mencionados. Finalmente, se descartaron estudios que se referían a revisiones, artículos teóricos o estudios duplicados, debido que no se tenía la intención de discutir teorías de la disciplina.

\section{Resultados}

Con la revisión de la base de datos se obtuvieron 2169 referencias. Un gran porcentaje de registros estaban duplicados, no cumplían con alguno de los cuatro tópicos de selección o se trataba de estudios realizados en ecosistemas marinos. Considerando solo los criterios de selección, se obtuvo una base depurada de 217 trabajos en cualquiera de los cuatro tópicos en cuestión. Fue notorio un incremento en el número de publicaciones en los últimos años, particularmente en el periodo 2011-2018, donde se concentra $90 \%$ de los trabajos. Entre ellos destaca el 2015, a partir de este año se identifica el mayor aumento en el número de publicaciones anualmente. Sin embargo, es evidente que solo $10 \%$ de las publicaciones se realizaron durante 2000 y 2010 (Fig. 1).

La mayoría de las publicaciones corresponden a trabajos en el tópico de conectividad y planificación de la restauración (36\%) y en el tópico de modelado y planificación de conectividad de la cobertura vegetal/uso del suelo (29\%); seguidos por $14 \%$ en el tópico de conectividad en servicios ecosistémicos. Los trabajos publicados con el tópico de conectividad y cambio climático fueron escasos, representando solo $5 \%$ de la base de datos. Asimismo, también se identificaron publicaciones, de las cuales se realizaron estudios utilizando tres tópicos simultáneamente (Fig. 2). Por ejemplo, a la combinación del tópico en modelado y planificación de conectividad de la cobertura vegetal/uso del suelo con el tópico de conectividad de la planificación de la restauración, correspondió $13 \%$ de los trabajos considerados. Para la combinación de conectividad en servicios ecosistémicos, con el tópico de conectividad y cambio climático, 2\%. Y para la combinación de conectividad de la planificación de la restauración, con el tópico de conectividad y cambio climático, 1\%.

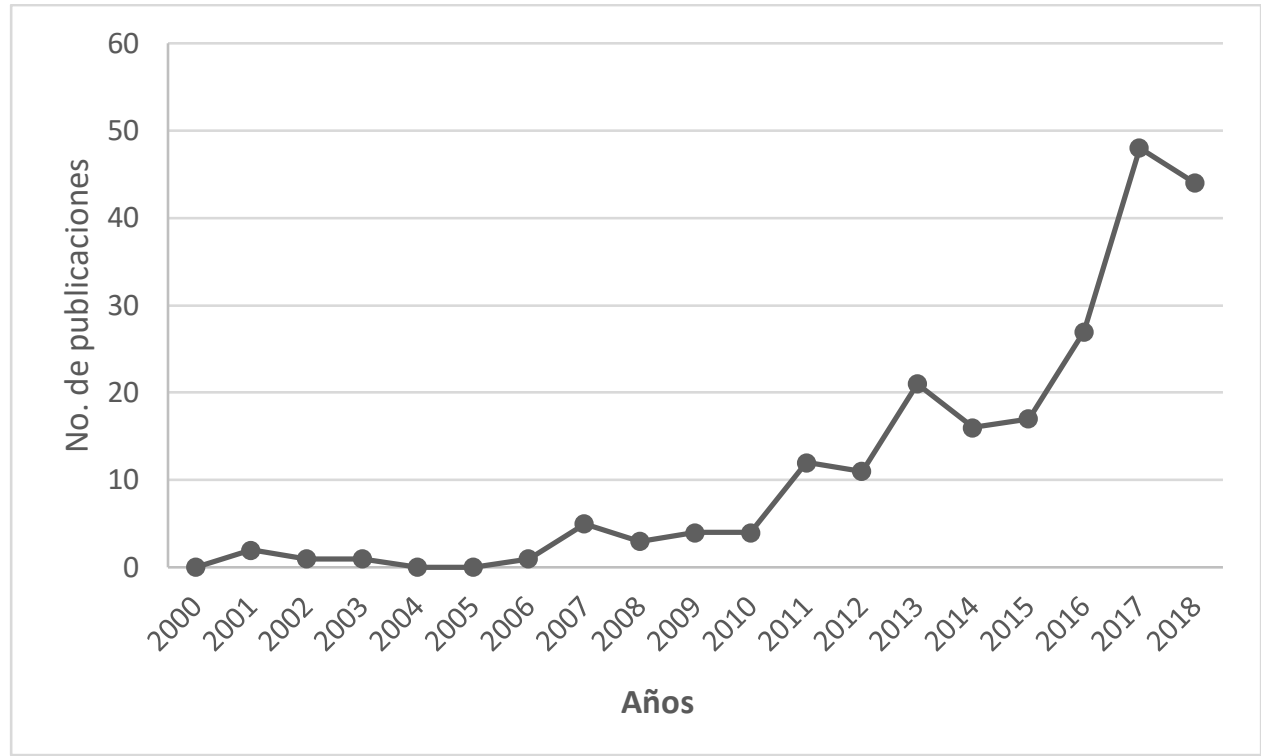

FIGURA 1. Número de publicaciones por año. 


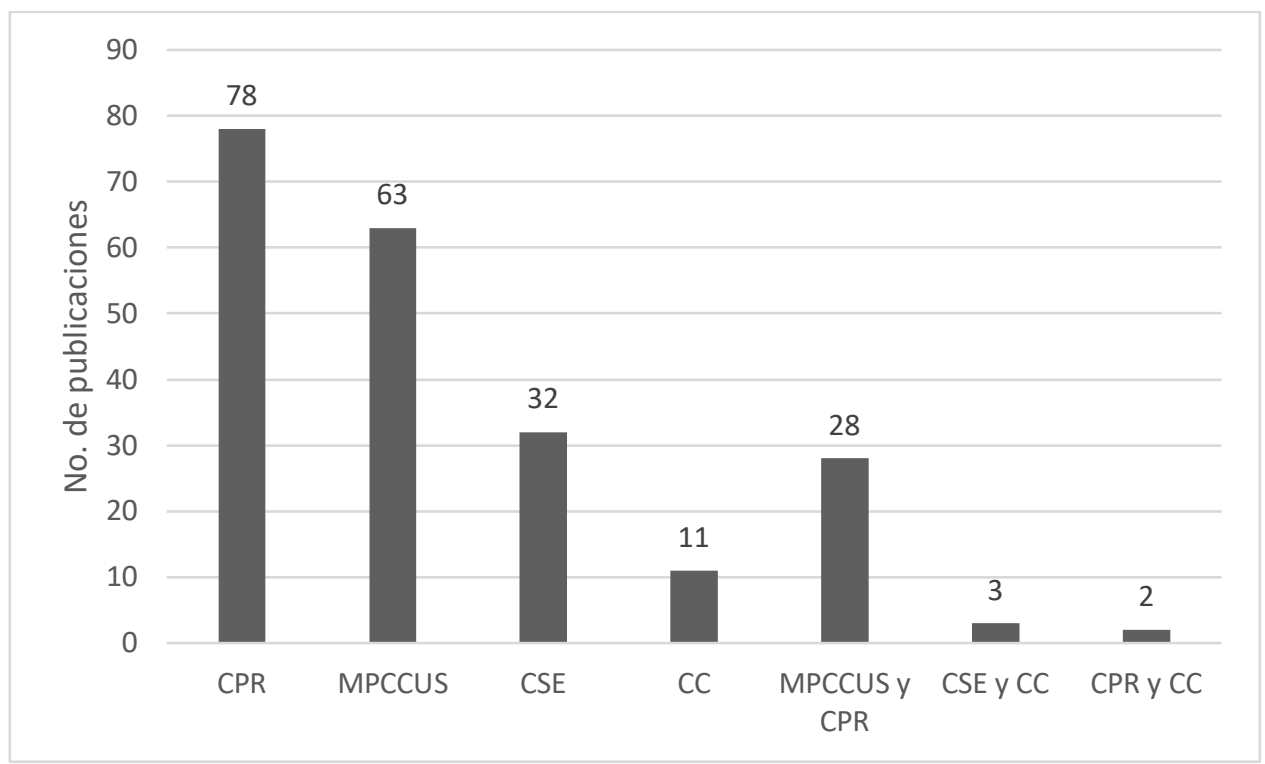

FiguRA 2. Número de trabajos realizados (2000-2018) en cada uno de los tópicos en países de América Latina.

CPR (conectividad a la planificación de la restauración), MPCCUS (modelado y planificación de conectividad y cobertura vegetal/uso del suelo), CSE (conectividad en servicios ecosistémicos) y CC (conectividad y cambio climático).

En solo 14 países de América Latina se han realizado estudios de conectividad del paisaje, aplicando uno o dos de los cuatros tópicos en cuestión. Entre ellos destacan Brasil, México, Argentina, Chile y Colombia con el mayor porcentaje del total de publicaciones realizadas con $83 \%$. Bolivia, Costa Rica, Guatemala y Perú concentraron 11\%; mientras que en Ecuador, El Salvador, Nicaragua, Paraguay y Venezuela solo registraron 6\%, siendo los países con las menores cantidades de trabajos realizados (Fig. 3). Asimismo, en la tabla 1 se muestra una radiografía amplia de los números de trabajos identificados para cada país, de acuerdo con el tópico analizado.

Con respecto al número de revistas identificadas destacan cuatro de ellas, las cuales concentran el mayor número e interés por publicar los resultados obtenidos orientados a la conectividad del paisaje en cualquiera de los cuatro tópicos en cuestión, tal como se muestra en la figura 4. En este sentido, también se lograron identificar siete tipos de cubiertas vegetales (Fig. 5), siendo Brasil el país que destaca por tener trabajos en seis de los siete biomas identificados en este estudio, particularmente cuenta con el mayor número de trabajos realizados para los bosques tropicales y templados con 36 y 22 estudios respectivamente. Seguido por México, en el cual se elaboraron estudios en siete biomas, principalmente en bosques tropicales (21 artículos) y en bosques templados con (ocho artículos). Por su parte, en Argentina se elaboraron trabajos en cuatro biomas, principalmente en bosques tropicales y en bosques templados.

De acuerdo con el contenido de los grupos taxonómicos (familias, géneros y especies) más representativos para el análisis realizado en los países de América Latina, se identificó que $77 \%$ de los estudios focales correspondieron a seis clases, 24 órdenes, 67 familias y 137 especies. Las tres clases más frecuentes fueron vertebrados: mamíferos (78 artículos), aves (67 artículos) e insectos (18 artículos). El orden más estudiado fue el de Carnívoros (62 artículos). La familia de los felinos es frecuentemente usada en los análisis de conectividad (47 artículos), mientras que la especie más estudiada fue Panthera onca (jaguar, 19 artículos). Cabe resaltar que en 38 estudios no utilizaron especies focales. 


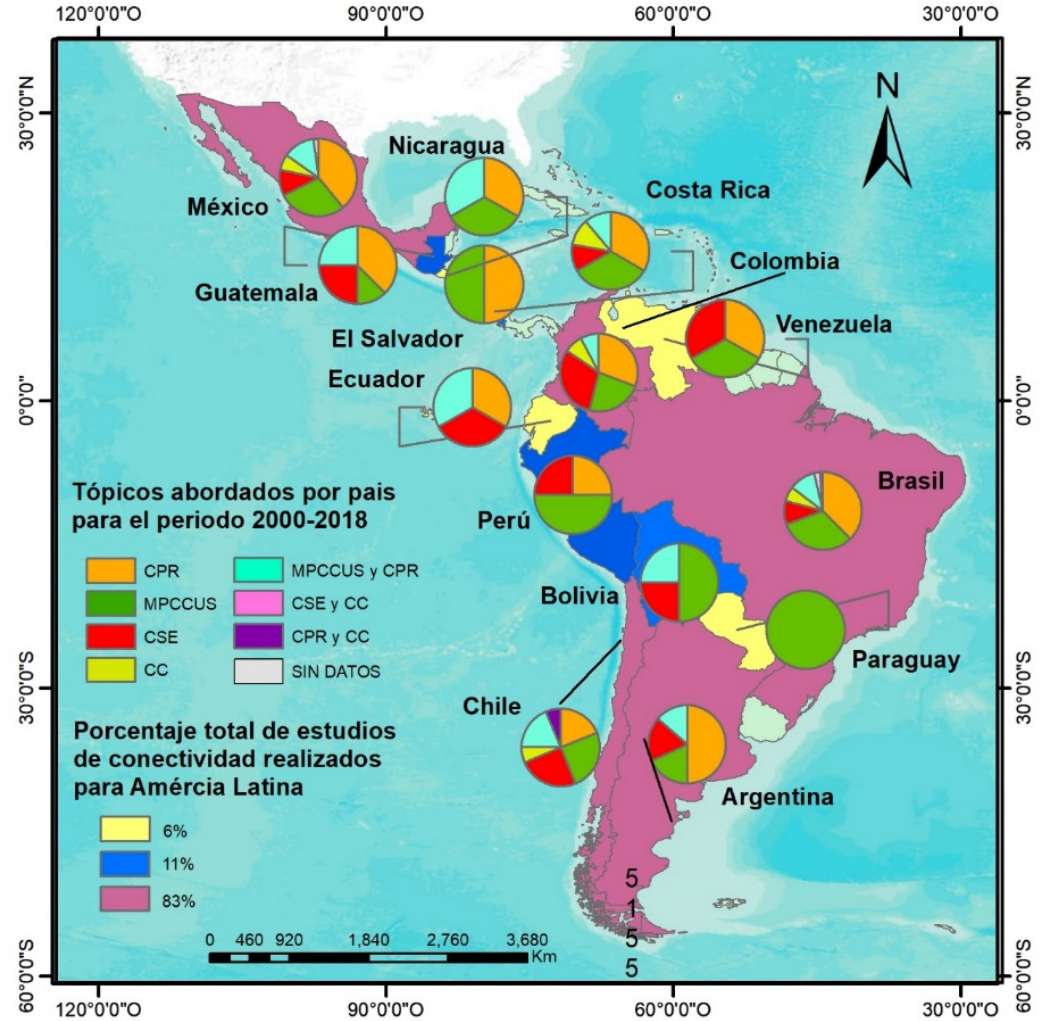

FIGURA 3. Mapa para cada tópico abordado en países de América Latina y porcentaje total de estudios realizados. CPR (conectividad a la planificación de la restauración), MPCCUS (modelado y planificación de conectividad y cobertura vegetal/uso del suelo), CSE (conectividad en servicios ecosistémicos) y CC (conectividad y cambio climático).

TABLA 1. Número de trabajos publicados (2000-2018) en cada uno de los países de América Latina.

\begin{tabular}{lcccccccc}
\hline Tópicos/Países & CPR & MPCCUS & CSE & CC & $\begin{array}{c}\text { MPCCUS y } \\
\text { CPR }\end{array}$ & $\begin{array}{c}\text { CSE y CC } \\
\text { CPR y CC }\end{array}$ & Total \\
\hline Brasil & 31 & 26 & 8 & 5 & 9 & 2 & 1 & 82 \\
México & 18 & 13 & 5 & 3 & 6 & 1 & 0 & 46 \\
Argentina & 11 & 4 & 4 & 0 & 3 & 0 & 0 & 22 \\
Chile & 3 & 4 & 4 & 1 & 3 & 0 & 0 & 16 \\
Colombia & 4 & 3 & 4 & 1 & 1 & 0 & 0 & 13 \\
Costa Rica & 3 & 3 & 1 & 1 & 1 & 0 & 0 & 9 \\
Guatemala & 3 & 1 & 2 & 0 & 2 & 0 & 0 & 8 \\
Bolivia & 0 & 2 & 1 & 0 & 1 & 0 & 0 & 4 \\
Perú & 1 & 2 & 1 & 0 & 0 & 0 & 0 & 4 \\
Ecuador & 1 & 0 & 1 & 0 & 1 & 0 & 0 & 3 \\
El Salvador & 1 & 1 & 0 & 0 & 1 & 0 & 0 & 3 \\
Paraguay & 0 & 2 & 0 & 0 & 0 & 0 & 0 & 2 \\
Venezuela & 1 & 1 & 1 & 0 & 0 & 0 & 0 & 3 \\
Nicaragua & 1 & 1 & 0 & 0 & 0 & 0 & 0 & 2 \\
Total & 78 & 63 & 32 & 11 & 28 & 3 & 2 & 217 \\
\hline
\end{tabular}

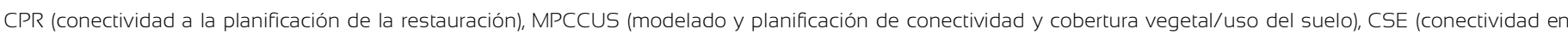
servicios ecosistémicos) y CC (conectividad y cambio climático). 


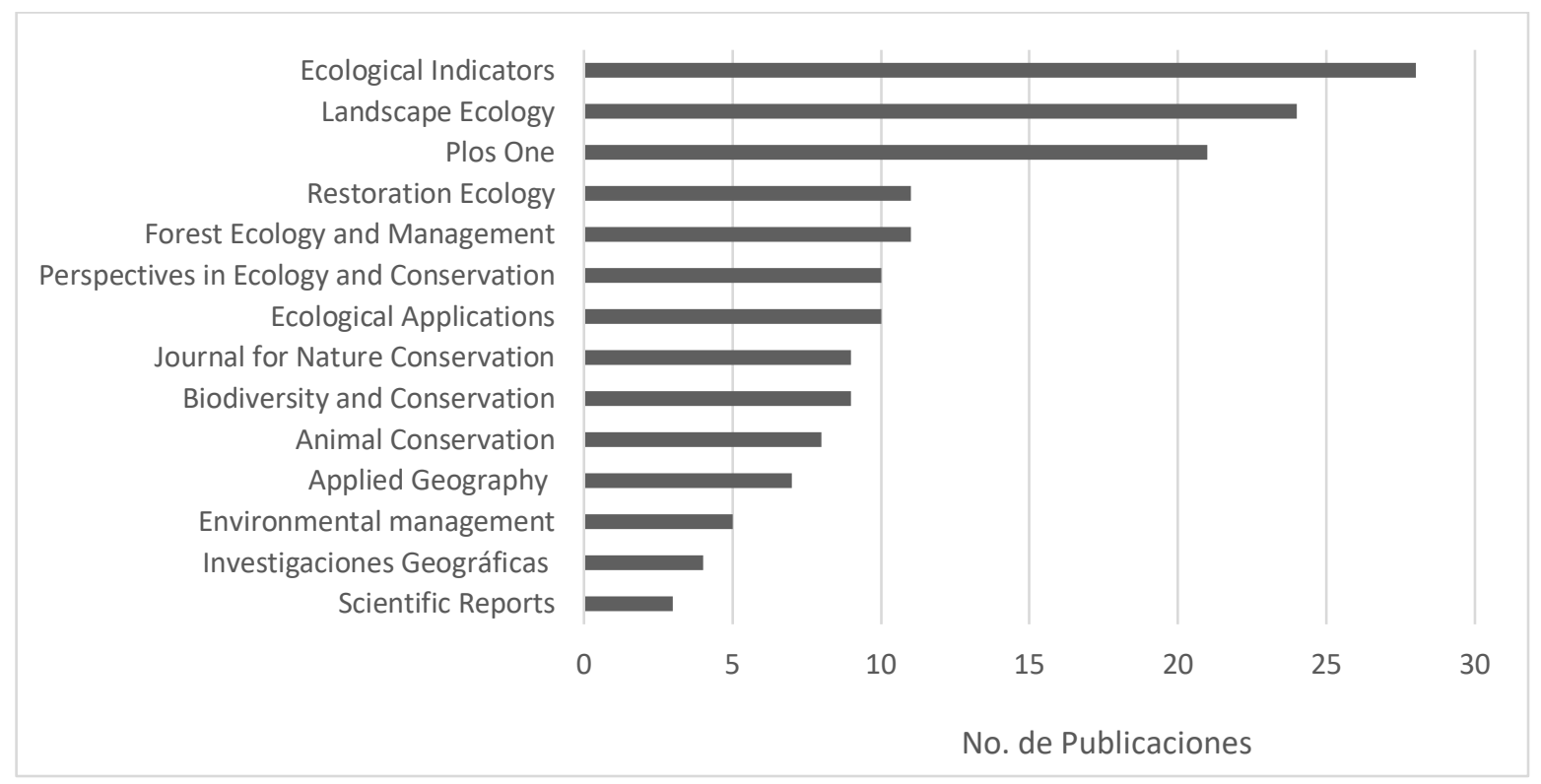

FIGURA 4. Número de trabajos publicados entre 2000 y 2018 en diferentes revistas de acuerdo con la base de datos consultada.

Solo se muestran las revistas que cuentan con mayor número de publicaciones.

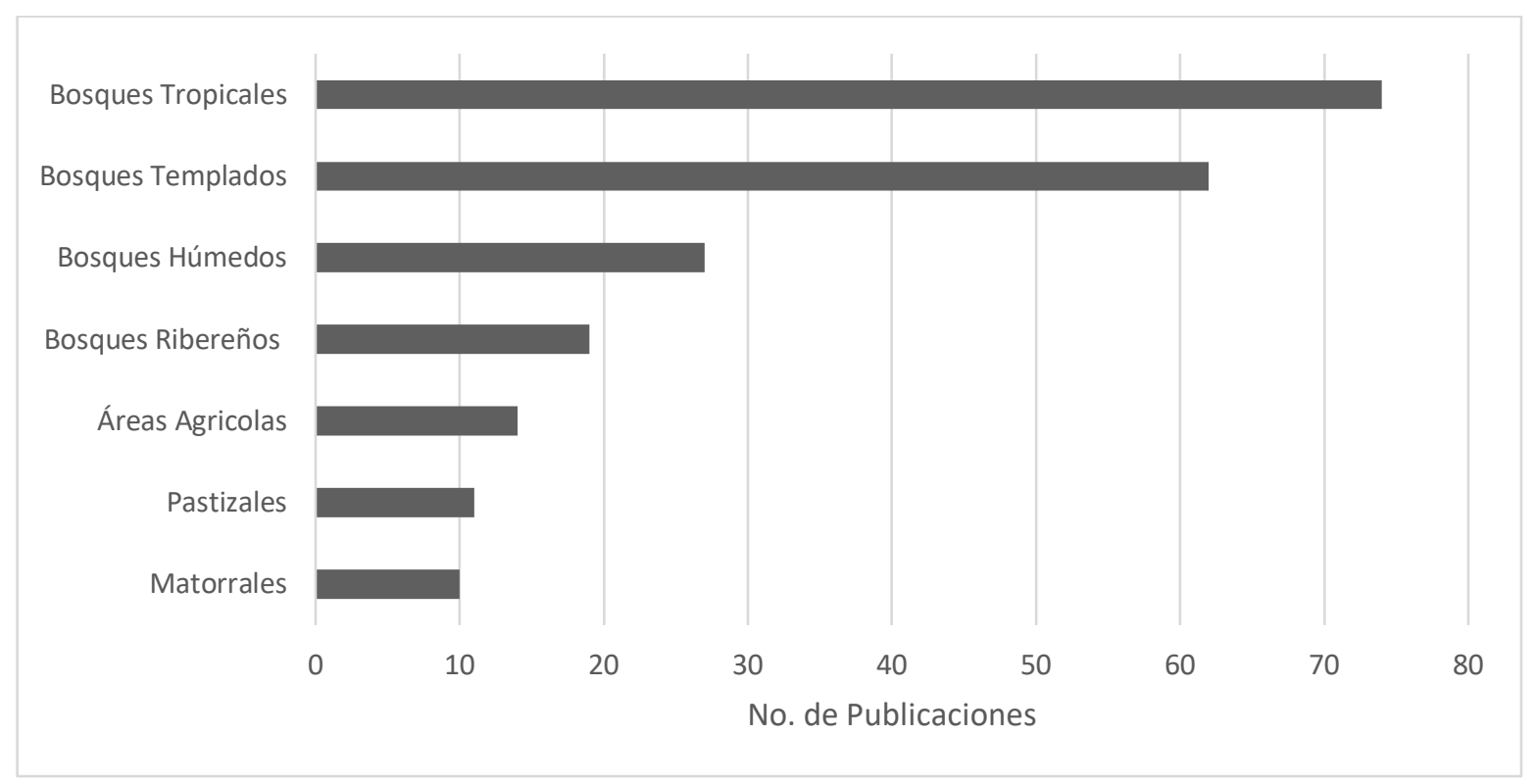

FIGURA 5. Número de artículos y coberturas vegetales/usos del suelo que se identificaron para América Latina. 
Se identificaron 16 objetivos, acciones o propuestas para los cuatro tópicos en cuestión (Fig. 6). Los más frecuentes fueron: a) identificación de áreas para restauración con 53 artículos, b) conectividad funcional y genética con 42 artículos, c) análisis del cambio en el uso del suelo con 36 artículos, d) evaluación de áreas naturales protegidas y áreas prioritarias para la conservación con 14 artículos, e) evaluación y estado de conservación del paisaje con 11 artículos, f) conectividad de acciones para la gestión con nueve artículos, g) modelado de distribución potencial con ocho artículos y f) análisis de efecto de borde con siete artículos. Con base en estos objetivos, acciones o propuestas identificadas, se emplearon 19 diferentes tipos de métodos para los cuatro tópicos en cuestión de los 14 países analizados (Fig. 7).

\section{DISCUSIÓN}

América Latina es la región con mayor porcentaje de bosques tropicales con 58.1\% (Forest Resources Assessment [FRA], 2015), cuenta con la mayor cantidad de biodiversidad en el mundo y amplias áreas de reservas de sumideros de carbono (Houghton, 2007; Eva et al., 2012).
Sin embargo, desde el siglo XX y a principios del actual ha registrado pérdidas en sus superficies originales a través de la expansión de la frontera agrícola (Laurance et al., 2011; Corlett, 2015; Leija y Pavón, 2017). Su conversión se debe principalmente a su alto potencial de productividad agrícola (Hansen et al., 2013), causa principal de la pérdida de vastas extensiones de bosques y sabanas en los trópicos, con altas tasas de deforestación (Wright, 2005; Kim et al., 2015).

La incorporación de América Latina al comercio global, la urbanización a través del aumento exponencial de la población y el consumo per cápita, son algunos de los impulsores clave de la deforestación tropical (DeFries et al., 2010). Además, la falta de oportunidades y el escaso desarrollo económico en la región han generado desigualdad y segregación social por la falta de estrategias inclusivas y participativas que permitan obtener una mejor calidad de vida para la población (Kacowicz, 2008). Estos impactos directos e indirectos en los patrones de uso del suelo a múltiples escalas son un ejemplo de los conductores de la transformación de las coberturas naturales (Geist y Lambin, 2001; Lambin et al., 2003).

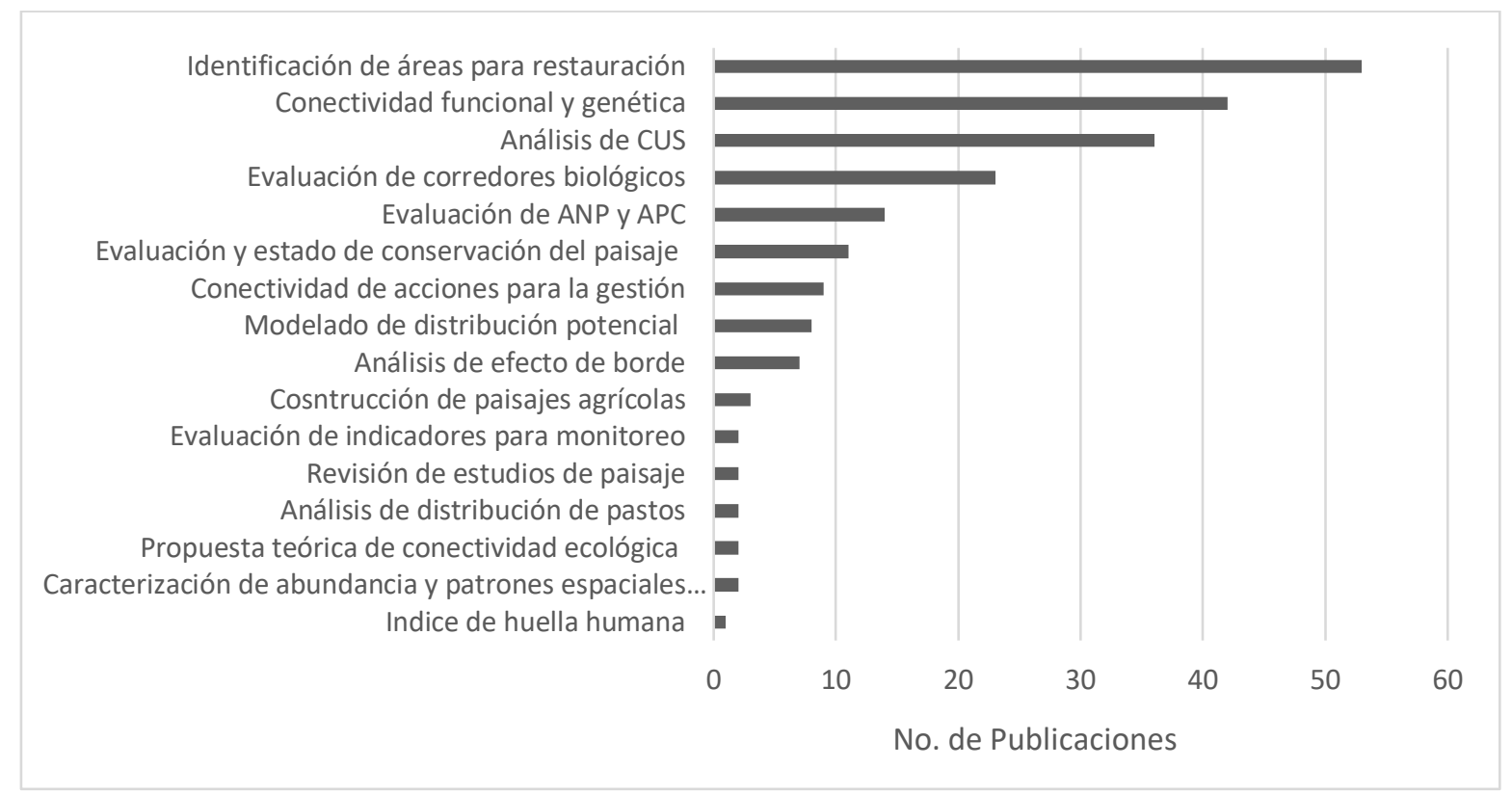

FiguRA 6. Objetivos, acciones o propuestas para los estudios de conectividad en América Latina en cualquiera de los cuatros tópicos en cuestión. 


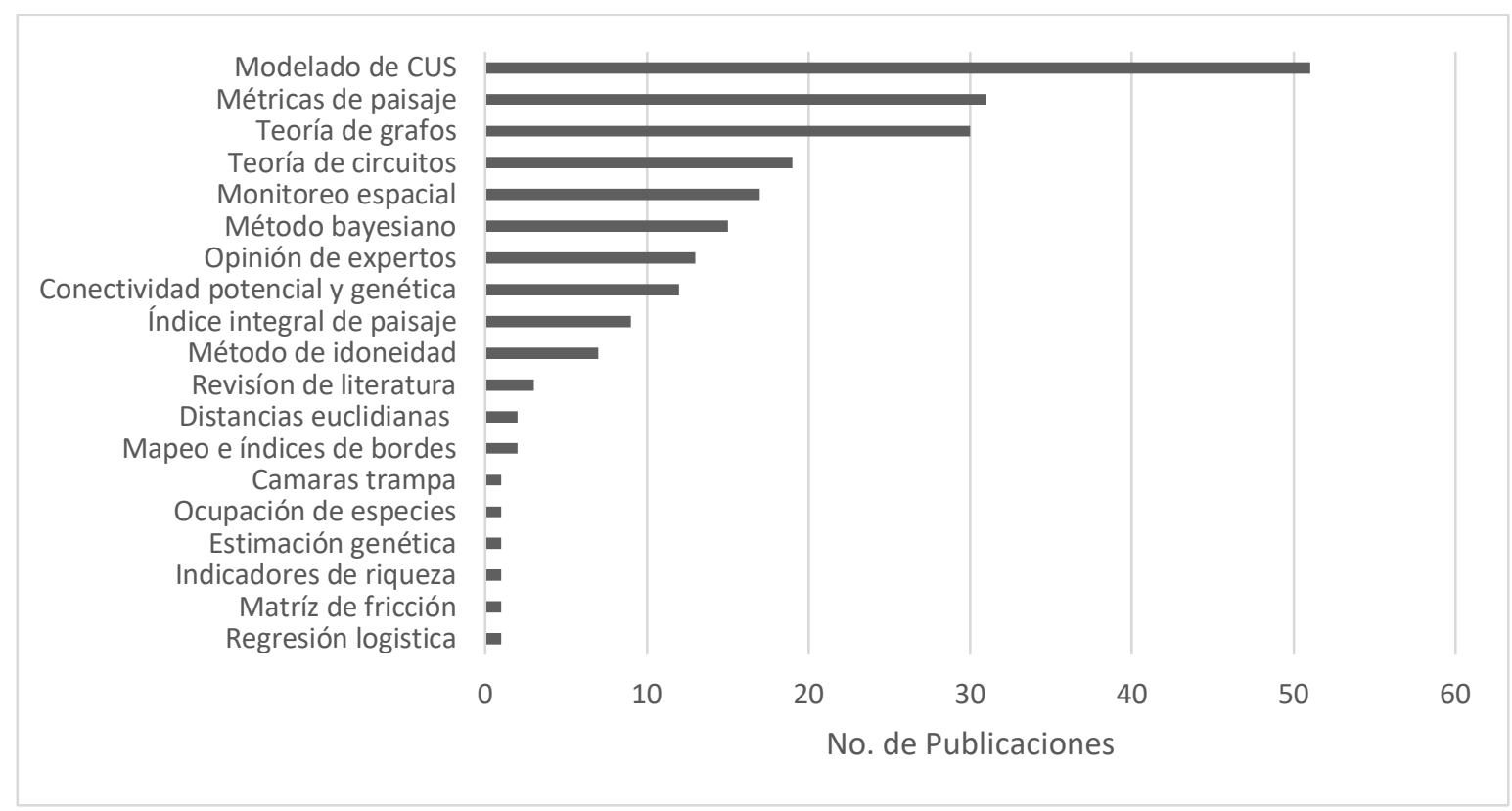

FIgURA 7. Tipo de métodos empleados para los cuatro tópicos en cuestión en países de América Latina.

La deforestación es un fenómeno que sigue siendo un tema importante en la actualidad, debido a que proporciona datos elementales sobre los impulsores del cambio en el uso del suelo (Sohoulande, 2017). Por ello es importante, comprender sus patrones y las causas a mayor detalle, las cuales permitan proponer de acciones para la conservación de áreas sensibles o ecológicamente valiosas y frenar la transformación y fragmentación de los bosques en la región (Martins et al., 2012; Newbold et al., 2015; Clark y Tilman, 2017). Una de estas acciones clave para la conservación es la conectividad del paisaje; su diseño y aplicación a diferentes escalas permitiría comprender los procesos ecológicos en paisajes fragmentados, como la interacción, el movimiento y la supervivencia de las especies, además de los elementos de paisaje que contribuyen a la conectividad estructural y funcional (Opdam et al., 2003; Coulon et al., 2004; Mitchell et al., 2013). Sin embargo, la funcionalidad de esta acción clave está supeditada a múltiples factores, lo que a su vez se refleja en impactos y limitaciones como el cambio en el uso del suelo (Luque et al., 2012).

A pesar de los desafíos y dificultades que implica llevar a cabo estudios orientados a la conectividad del paisaje en
América Latina, en 18 años (2000-2018) se ha identificado un aumento significativo de trabajos clave sobre conectividad en 14 de los 20 países que conforman la región. Actualmente, y en general, la toma de decisiones y las acciones en pro de la conservación de los ecosistemas terrestres tienen mayor fundamento científico (Townshend et al., 2012; Hansen et al., 2013; Pettorelli et al., 2014). Principalmente a una mejor comprensión de los procesos ecológicos y a la generación de nueva información, así como de los avances en tecnologías geoespaciales y capacidades de cómputo, las cuales permiten la generación, procesamiento y análisis de grandes volúmenes de datos.

Sin embargo, es preocupante que para los seis países restantes no se logró identificar evidencias de haberse realizado algún tipo de trabajo sobre conectividad del paisaje bajo los cuatro tópicos en cuestión. No hay una explicación contundente de por qué Uruguay, Republica Dominicana, Puerto Rico, Cuba y Panamá no cuenten con trabajos realizados. Se asevera la posibilidad de que estos países tengan problemas sociales, económicos y políticos que impidan el desarrollo de investigación científica orientada a la conservación de los ecosistemas terrestres, 
particularmente en estudios orientados a la conectividad del paisaje con enfoque para los cuatro tópicos (Carabias et al., 2007; Ceccon et al., 2015). Los tópicos con mayor número de estudios fueron: conectividad a la planificación de la restauración, modelado y planificación de conectividad y cobertura vegetal/uso del suelo y conectividad en servicios ecosistémicos, destacando los países de Brasil, México, Argentina y Colombia, los cuales concentran el mayor número de trabajos. También se identificó la combinación en el uso de estos tres tópicos solo para Brasil, México y Chile que fueron los que más estudios abordaron simultáneamente; esto indica que existe un creciente interés de investigación en estos temas.

\section{Tópico conectividad y planificación de la restauración (CPR)}

El interés por trabajos orientados a la restauración ecológica ha incrementado significativamente y ha tomado un mayor auge en los últimos años. A partir de la creación de la Society Ecological Restoration (SER) en 2004, el número de publicaciones y revistas especializadas en el tema ha aumentado considerablemente (Armesto et al., 2007; Sarukhán, 2007; Bonfil et al., 2015). Sin embargo, los trabajos desarrollados bajo el enfoque de conectividad a la planificación de la restauración aún son incipientes, a pesar de ser el tópico con el mayor número total de estudios realizados para esta región con 36\%. En este trabajo se postula que la carencia de este tipo de trabajos se debe principalmente al contexto socioeconómico e histórico de AL, el cual dimensiona un desafío importante para los especialistas en restauración, principalmente por implicaciones directas de enfoques teóricos y prácticos (Suding et al., 2004; Kacowicz, 2008; DeFries et al., 2010). Brasil, México y Argentina registraron el mayor número de publicaciones referentes a este tópico. Las principales coberturas terrestres estudiadas son los bosques tropicales, los templados y, en menor medida, las selvas. Lo que permite suponer y asociar la preocupación en el ritmo acelerado de la deforestación es estas áreas, cuya complejidad en riqueza y diversidad biológica las clasifica como ecorregiones críticas o en peligro, a pesar de los avances de legislación ambiental y programas de conservación para su protección (Carabias et al., 2007; Leidner y Haddad, 2011; Parks et al., 2013; Martínez-Ramos et al., 2016). Asimismo, organizaciones internacionales, nacionales, países y diferentes actores políticos se han sumado a la causa en la promoción de acciones e iniciativas de restauración forestal a escala mundial, con la finalidad de lograr los objetivos de la Declaración de Nueva York (Laestadius et al., 2011; Clereci et al., 2018).

Además, se requiere un enfoque de planificación multidisciplinario, que permita la implementación de una restauración rentable en términos de costos y resultados (Birch et al., 2010; Tambosi y Metzger, 2013; Sayer et al., 2013). Por ejemplo, el enfoque basado en la distancia de menor costo y la teoría de circuitos permitirían la identificación de áreas con potencial para la conectividad estructural y funcional del paisaje con la finalidad de priorizar sitios para restauración y conservación (McRae, Hall y Beier, 2012). Sin embargo, estas acciones y enfoques basados en ciencia, políticas públicas y limitaciones emergentes representan un desafío importante para lograr el compromiso de una restauración global (Laestadius et al., 2011; Suding et al., 2015; Brancalion y van Melis, 2017).

\section{Tópico modelado y planificación de conectividad de la cobertura vegetal/uso del suelo (MPCCUS)}

Los esfuerzos por llevar a cabo estudios orientados al análisis y modelado de los procesos de cambio en el uso del suelo, a finales del siglo $\mathrm{XX}$, se han abordado principalmente desde el punto de vista espacial, tal como lo describen Turner y Gardner (1990), Elston y Bucland (1993), Lambin (1994). Los trabajos se han enfocado particularmente en responder las preguntas ¿por qué ocurre el cambio en el uso del suelo? ¿dónde? y ¿cuándo ocurre el cambio? Por lo que suelen ser muy descriptivos, con la finalidad de conocer la dinámica espacio-temporal y el ritmo de las tasas de deforestación, a través de herramientas metodológicas en sensores remotos (Lambin, 1997). 
En las últimas décadas, se ha evidenciado un avance significativo en el incremento de este tipo investigaciones. En América Latina, las publicaciones relacionadas con el tópico de modelado de cambio en el uso del suelo con énfasis en la conectividad del paisaje corresponden a $29 \%$. Brasil y México son los países que concentran la mayoría de las publicaciones con 18\%. Por su parte, Argentina, Chile, Colombia y Costa Rica representan tan solo 6\% de trabajos para este tópico.

Destaca la calidad de la información, el progreso reciente en la disponibilidad y potencia de datos, así como las herramientas metodológicas utilizadas, las cuales han tenido un desarrollo paralelo a la información geográfica para ser integrada en el modelado y planificación de conectividad de la cobertura vegetal y uso del suelo (Kim et al., 2015; Oliveira et al., 2019). Por ejemplo, en estudios recientes se observa no solo una descripción espacial, sino un mayor desarrollo de análisis integral, como lo postulan Horta et al. (2018), Zimbres et al. (2017), Varga et al. (2016) y Castilho et al. (2015). Los avances resaltan la importancia de las herramientas actuales en los modelos de identificación de tendencias en el cambio en el uso del suelo. Los modelos proporcionan una base coherente y especialmente explícita para la inferencia en estudios de conectividad del paisaje, permitiendo desarrollar esfuerzos de conservación oportuna especialmente por procesos acelerados de la deforestación (Piquer-Rodríguez et al., 2012).

\section{Tópico conectividad en servicios ecosistémicos (CSE)}

Se considera que la relación y complementariedad entre los estudios de conectividad del paisaje y los servicios ecosistémicos es compleja (Millenium Ecosystem Assessment [MEA], 2005); sin embargo, permiten asociar la relación espacial entre unidades funcionales, los hábitats y los elementos del paisaje (MEA, 2005). En este tópico, la mayoría de los estudios se han elaborado en Brasil, México, Argentina, Chile y Colombia. Desafortunadamente ningún trabajo se realizó en El Salvador, Paraguay y Nicaragua. Las principales acciones y tipos de coberturas identificados se relacionan con: a) la conectividad estructural y genética para diferentes especies focales en selvas tropicales (Moraes $e t$ al., 2018); b) identificación de sitios clave para la conectividad de poblaciones en mamíferos en bosques (Diniz et al., 2017); c) actualización de áreas naturales protegidas para la conservación de plantas y fauna en peligro de extinción en bosques (Cardoso da Silva et al., 2018); d) construcción de paisajes agrícolas para la conservación de mamíferos terrestres (Hardt et al., 2015).

Las tendencias identificadas de estos estudios proponen, desde la funcionalidad de los ecosistemas, investigaciones acerca de las numerosas interacciones que pueden ocurrir entre diferentes objetos de estudio (MartínLópez et al., 2012a; Maskell et al., 2013). Este tipo de trabajos proporciona o adopta los trade-offs, los cuales pueden ser desde temporales (los que implican beneficios y costos a largo plazo) hasta espaciales (generando un beneficio local, regional o global) (Martín-López y Montes, 2011). Cabe mencionar que los trade-offs, varían de acuerdo con la priorización de los servicios efectuados (MEA, 2005). Por ejemplo, la falta de conectividad del paisaje repercute en el intercambio, compensación y recuperación de flujos, debido a que no siempre son comparables. Por ello, es pertinente y necesario realizar acciones que permitan la conservación, restauración y uso sostenible de los ecosistemas para mantener sus servicios y funcionalidad.

\section{Tópico conectividad y cambio climático CCC}

La importancia en el mantenimiento de los ecosistemas como soporte para la distribución de los organismos es fundamental (Hannah et al., 2002). El aumento en la temperatura del planeta por el cambio climático ha generado que los patrones de biodiversidad se vean afectados directamente (Wan et al., 2014). El interés por comprender con mayor amplitud el efecto en la distribución potencial en diferentes especies, ha causado que especialistas en diversas áreas desarrollen investigaciones para entender la funcionalidad de los ecosistemas terrestres a través de la conectividad del paisaje con métodos y 
enfoques que respaldan su evaluación (Hoglund, 2009; Rayfield et al., 2011).

En la actualidad, el cambio climático y sus impactos siguen siendo temas incipientes, debido a las incertidumbres asociadas con los datos existentes (Loyola et al., 2012; Garcia et al., 2014). Los estudios realizados e identificados en este tópico fueron los menos representados, de acuerdo con los resultados obtenidos con tan solo 5\%. En Brasil y México se desarrollaron cinco y tres trabajos, respectivamente. Los principales enfoques que se han abordado en este tópico fueron: a) implicaciones del cambio climático y las actividades humanas sobre la conectividad entre parches, los cuales apoyan la identificación de áreas prioritarias y corredores potenciales para la conservación del elefante (Zacarias y Loyola, 2018); b) el impacto de la distribución espacial de manchones de pastos en la diversidad de macrofauna y temperatura del suelo en pasturas (Mathieu et al., 2009); c) la evaluación de los corredores biológicos como una posible medida de adaptación ante el cambio climático (Imbach et al., 2013); d) la incorporación de enfoques de planificación sistemática para la conservación de la biodiversidad por el cambio climático (Groves et al., 2011).

Los trabajos anteriormente mencionados muestran las diferentes aplicaciones en pro de mejorar la conectividad funcional basada en especies focales y ecosistemas poco comunes como pastizales y matorrales en respuesta ante los efectos del cambio climático. Estos enfoques y acciones han permitido comprender cómo responderán las especies por el aumento de la temperatura del planeta. Los estudios mencionados ponen énfasis en la combinación de datos sobre observaciones de especies y variables ambientales con la finalidad de identificar áreas con condiciones climáticas apropiadas para las especies (Summers et al., 2012; Brown y Yoder, 2015).

A pesar de estos avances y de los pocos trabajos registrados para este tópico, es probable que muchas de las especies y ecosistemas para los que se desarrollen planes de conservación enfrenten tensiones cada vez mayores debido a los efectos directos e indirectos del cambio climático (IPCC, 2007). Pero lo esfuerzos, espacialmente extensos, para mejorar la conectividad a lo largo de los principales gradientes ambientales y el monitoreo riguroso de las respuestas de las especies coadyuvarán a desarrollar futuras acciones de manejo que proporcionarán una base sólida para la conservación a medida que aumentan las temperaturas (Krosby et al., 2010).

\section{Oportunidades y perspectivas}

La diversidad sociocultural y ecológica de América Latina brinda oportunidades para generar nuevos enfoques en el aprovechamiento y manejo de los recursos naturales para el desarrollo de acciones futuras en la planeación ambiental (Armesto et al., 2007). Los efectos de la deforestación, derivados a de impactos antropogénicos y de la liberación de mercados nacionales en América del Norte (México), Central y Sur, han generado que grandes áreas ocupadas por ecosistemas terrestres se hayan transformado desde el siglo XX, principalmente por la agricultura (Santos, 2010), además de la inserción de políticas agrarias y tecnificación agrícola para la exportación de productos primarios, lo que ha generado la expansión de nuevas tierras para su desarrollo (Furtado, 2007). Lo anterior ha promovido la creación de objetivos o acciones de planeación que permitan la aplicación de estrategias, herramientas metodológicas y enfoques multidisciplinarios para la conservación de los ecosistemas, basada principalmente en mejorar la restauración ecológica y conectividad del paisaje (Foley et al., 2005).

La conectividad del paisaje representa grandes desafíos y oportunidades, que solo se pueden lograr y fortalecer a través de enfoques multidisciplinarios que complementen la teoría y la aplicación sensu stricto, dentro de un entorno cultural, político y biológico que prevalece en América Latina (Armesto et al., 2007). Los documentos registrados en este estudio resaltan la importancia de llevar a cabo un mayor número de trabajos en cualquiera de los cuatro tópicos en cuestión. A pesar de que solo en 14 países se ha realizado algún tipo de estudio sobre conectividad del paisaje, particularmente asociado al tópico de planificación de la restauración y al de modelado y planificación de 
conectividad de la cobertura vegetal/uso del suelo, los trabajos se han realizado principalmente en Brasil y México (82 y 46 respectivamente); esto refleja una mayor capacidad de divulgación para estos dos países. Adicionalmente, esta tendencia en la productividad se asocia a los mecanismos de cooperación internacional y nacional para realizar estudios enfocados en el campo de la conectividad del paisaje.

Por ejemplo, el Corredor Biológico Mesoamericano, conformado por siete países de Centro América y México, estaba enfocado en un modelo integral destinado a detener la expansión de la frontera agropecuaria, para proteger remanentes de selvas y bosques que permitieran hacer frente al fenómeno de cambio climático. El principal objetivo era disminuir la fragmentación y mejorar la conectividad del paisaje de los ecosistemas, promoviendo los procesos productivos sustentables que mejoran la calidad de vida de las poblaciones locales para conservar la diversidad biológica (Banco Mundial, 2001; Chang et al., 2001). Otro ejemplo es el realizado a través del Convenio sobre la Diversidad Biológica en 2010, con base en el Objetivo Aichi 11, el cual tenía la intención de tomar medidas efectivas y urgentes para detener la pérdida de diversidad biológica, asegurando que para 2020 los ecosistemas fueran resilientes y siguieran suministrando servicios esenciales. Esto permitiría asegurar la variedad de la vida del planeta y contribuir al bienestar humano y a la erradicación de la pobreza (Convention on Biological Diversity [CDB], 2010). En la convención se acordó que $17 \%$ de la tierra se conectara por sistemas de áreas protegidas para el año 2020 (Meta 11 de Aichi). Saura et al. (2019) documentaron, a través de una evaluación global para todos los países, las tendencias en la conectividad de áreas protegidas terrestres cada dos años desde 2010 hasta 2018. Estos autores identificaron un aumento en la conectividad de las áreas protegidas para América del Sur y del Caribe, destacando países como Brasil, Costa Rica, Guayana y República Dominicana que han cumplido con el objetivo de la Meta 11 de Aichi en incrementar la conectividad de sus áreas protegidas (Saura et al., 2017). Asimismo, se encuentra en desarrollo el proyecto de conectividad ecorregional en los países andinos tropicales conformado por cinco países en Sudamérica, cuyo propósito es similar al objetivo de Aichi (Castillo et al., 2019).

Por su parte, Armesto et al. (2007) conformaron una red de ecológica latinoamericana enfocada en la restauración, lo cual podría explicar la mayor cantidad de trabajos para el tópico de CPR. Entre los principales objetivos de la red se encuentra : 1) promover la comunicación entre los científicos y otros profesionales que trabajan en proyectos de restauración, rehabilitación o reforestación dentro de la región; (2) transmitir a los miembros de la red el conocimiento de la amplia diversidad de ecosistemas terrestres y acuáticos; (3) estimular el pensamiento innovador y los avances conceptuales en la ciencia de la restauración para enfrentar el desafío de escenarios culturales y ecológicos particulares en América Latina; (4) fomentar la creación de capacidad local y la educación del público en general en la ciencia de la restauración; y (5) estimular la transferencia de conocimientos técnicos a las autoridades gubernamentales a cargo de la planificación del territorio.

En cuanto a la identificación de especies focales en este trabajo, los resultados obtenidos tuvieron una semejanza a los registrados en estudios previos (Zeller et al., 2012; Correa-Ayram et al., 2016). Los carnívoros, como el jaguar y el puma o león de montaña (Puma concolor) han marcado, entre los investigadores, una enorme preferencia sobre estudios donde predominan dichas especies focales carnívoras, por ser exigentes en relación con su área de distribución (Cardillo et al., 2004; Beier et al., 2008). Estos mamíferos son considerados los felinos más grandes del continente americano, por ser enormes depredadores, ocupar grandes extensiones de territorios y conformar la parte alta de la cadena alimenticia; sin embargo, también están sujetos a la persecución humana directa. Setenta y siete de cada cien estudios se concentró en especies focales y, de estos, solo $21 \%$ utilizó más de una especie en el análisis de conectividad.

Se considera que la concentración de los estudios identificados en los tópicos (CPR y MPCCUS) se debe a la comparación de las respuestas de las especies y a los 
impactos de los humanos en diferentes escalas espaciales. Lo anterior es importante no solo para evaluar su estado de conservación sino también para comprender las causas del declive o persistencia de las especies (Ciarniello et al., 2007; Liu et al., 2009). Las escalas espaciales juegan un papel clave en la consecución de los objetivos de la planificación ambiental y deben considerarse significativas en estudios de conectividad. Se identificó que, en cualquiera de los cuatro tópicos analizados, predominó la escala regional con $82 \%$ de los trabajos. En los trabajos analizados, las áreas de estudio no solo fueron acotadas por límites naturales, sino también políticos. Asimismo, la identificación de la escala espacial adecuada debe estar basada en los requisitos ecológicos, incluidos los procesos biofísicos que en ellas ocurren, lo que permitiría guiar los estudios enfocados a la conservación de la conectividad en cualquier de los cuatro tópicos en cuestión (Baguette y Van Dyck, 2007).

Finalmente, la conectividad del paisaje es un elemento esencial para la gestión y conservación de la biodiversidad. Su aplicación y desarrollo han llevado a realizar nuevos estudios que proporcionan una nueva forma de evaluar el impacto humano en la conectividad del paisaje a través del índice de huella humana, como una estrategia adaptativa para el cambio climático (Correa-Ayram et al., 2017; 2020). $\mathrm{Su}$ evaluación se puede aplicar a diferentes escalas y paisajes, sin embargo, la implementación de estos y otros estudios que aborden la conectividad del paisaje deben integrar nuevos enfoques, métodos e ideas de carácter multidisciplinario que, a su vez, desarrollen análisis holísticos. Por lo tanto, los avances en la investigación, mecanismos de colaboración y cooperación, sumados a los esfuerzos de los científicos, coadyuvarían a mejorar y comprender los paisajes modificados por causas de las actividades antropogénicas, con intención de mantener su conectividad y la interacción entre los organismos y el paisaje.

\section{CONCLUSIONES}

En América Latina, los estudios identificados sobre conectividad del paisaje en cualquiera de los cuatro tópicos en cuestión constituyen un primer acercamiento para orientar esfuerzos en la conservación de los ecosistemas. Sin embargo, también ofrecen una multitud de desafíos que obedecen a un modelo complejo de integraciones culturales, económicas, sociales, ambientales y políticas. A pesar de que se registró un limitado número de trabajos en cualquiera de los cuatro tópicos analizados, representan una importante colaboración científica, que proporciona una amplia gama de ejemplos y funcionamiento de sistemas complejos en diferentes regiones de América Latina en los últimos diez años. Aún quedan sesgos por resolver, particularmente en los seis países que no registraron esfuerzos para el desarrollo de estudios en conectividad del paisaje. Se identificaron escasos estudios realizados para los tópicos de conectividad en servicios ecosistémicos y de conectividad y cambio climático, particularmente para este último. Es evidente la necesidad de desarrollar un mayor número de investigaciones en los tópicos estudiados.

Finalmente, se destaca la existencia de mecanismos de cooperación internacionales para el diseño de estudios enfocados a la conectividad del paisaje en América Latina. Los cuales se han llevado a cabo a través de proyectos como el Corredor Biológico Mesoamericano, el Convenio sobre la Diversidad Biológica en 2010 con base en el objetivo Aichi 11 y el proyecto de conectividad ecorregional en los países andinos tropicales. Dichos proyectos buscan lograr que al menos $17 \%$ de las zonas terrestres, con particular importancia para la diversidad biológica y los servicios de los ecosistemas, se conserven por medio de sistemas de áreas protegidas administrados de manera eficaz y equitativa, ecológicamente representativa y bien conectada.

\section{RECONOCIMIENTOS}

El primer autor agradece el apoyo financiero y de investigación del Programa de Becas Postdoctorales DGAPA-UNAM. Asimismo, agradecemos la valiosa contribución de los comentarios y sugerencias de los revisores anónimos y del editor de la revista, los cuales han permitido mejorar el presente documento. 


\section{REFERENCIAS}

Armesto, J. J., Bautista, S., Del Val, E., Ferguson, B., García, X., Gaxiola, A., Godinez-Álvarez, H., Gann, G., López-Barrera, F., Manson, R., Núñez-Ávila, M., Ortiz-Arrona, C., Tognetti, P., \& WilliamsLinera, G. (2007). Towards in ecological restoration network: Reversing land degradation in Latin America. Frontiers in Ecology and the Environment, 5(4), 1-4. doi: 10.1890/15409295(2007)5[w1:TAERNR]2.0.CO;2

Baguette, M., \& Dyck, H. V. (2007). Landscape connectivity and animal behavior: Functional grain as a key determinant for dispersal. Landscape Ecology, 22, 1117-1129. doi: 10.1007/s10980-007-9108-4

Banco Mundial. (2001). Proyecto Corredor Biológico Mesoamericano. México: Oficina Regional para América Latina y el Caribe.

Beier, P., Majka, D. R., \& Spencer, W. D. (2008). Forks in the road: Choices in procedures for designing wildland linkages. Conservation Biology, 22(4), 836-851. doi: 10.1111/j.15231739.2008.00942.x

Bélisle, M. (2005). Measuring Landscape Connectivity: The challenge of behavioral landscape ecology. Ecology, 86(8), 1988-1995. doi: $\underline{10.1890 / 04-0923}$

Birch, J. C., Newton, A. C., Aquino, C. A., Cantarello, E., Echeverría, C., Kitzberger, T., \& Garavito, N. T. (2010). Cost-effectiveness of dryland forest restoration evaluated by spatial analysis of ecosystem services. PNAS, 107(50), 21925-21930. doi: $\underline{10.1073 / \text { pnas. } 1003369107}$

Bonfil, C., Fernández y Fernández, D., \& González-Espinosa, M. (2015). Un análisis preliminar del estado actual de los estudios de restauración ecológica en México. En B. Brown (Ed.), Tzintzuntzan, el lugar de los colibries - otra vez / Tzintzuntzan, place of the hummingbirds - again. Museo de Arte Contemporáneo Alfredo Zalce, Morelia (p. 28-39). Morelia, Michoacán: Editorial Estampas.

Brancalion, P. H. S., \& van Melis, J. (2017). On the need for innovation in ecological restoration. Annals of the Missouri Botanical Garden, 102(2), 227-236. doi: 10.3417/2016034

Brown, J. L., \& Yoder, A. D. (2015). Shifting ranges and conservation challenges for lemurs in the face of climate change. Ecology and Evolution, 5(6), 1131-1142. doi: 10.1002/ece3.1418

Carabias, J., Arriaga, V., \& Cervantes-Gutiérrez, V. (2007). Las políticas públicas de la restauración ambiental en México: limitantes, avances, rezagos y retos. Boletín de la Sociedad Botánica de México, 80 (Suplemento), 85-100. doi: 10.17129/botsci.1759

Cardillo, M., Purvis, A., Bielby, J., Mace, G.M., Sechrest, W., \& Gittleman, J. L. (2004). Human population density and extinction risk in the world's carnivores. PLoS Biology, 2(7), 909-914. doi: $\underline{10.1371 / \text { journal.pbio.0020197 }}$

Cardoso da Silva, J. M., Leal, I. R., \& Tabarelli, M. (2018). Caatinga: The largest tropical dry forest region in South America. Springer International Publishing. 482 pp. doi: 10.1007/978-3-319-68339-3

Castilho, C. S., Hackbart, V. C. S., Pivello, V. R., \& dos Santos, R. F. (2015). Evaluating landscape connectivity for puma concolor and panthera onca among atlantic forest protected areas. Environmental Management, 55(6), 1377-1389. doi: $\underline{10.1007 / \text { s00267-015-0463-7 }}$

Castillo, L. S., Correa-Ayram, C., Serrano, F., Chalán, L., Sánchez, F., More, A., Franco, O., Álvarez, J., Blomfield, H., Aguilera, V., Rivadeneira, C., Papadakis, J., Cárdenas, J. J., Morón, V., Yerena, E., Kroner, R. G., Matallana, C., Corzo, G., Areiza, A., González, R., \& Godinez, O. (2019). Cobertura de áreas protegidas y conectividad ecorregional en los países andinos tropicales. III Congreso de Áreas Protegidas de Latinoamérica y el Caribe.

Ceccon, E., Barrera-Cataño, J. I., Aronson, J., \& Martínez-Garza, C. (2015). The socioecological complexity of ecological restoration in Mexico. Restoration Ecology, 23(4), 331-336. doi: $\underline{10.1111 / \mathrm{rec} .12228}$

Chang, E., Echeverría, J., \& Miller y Tunstall, D. (2001). Acciones catalíticas para implementar el Corredor Biológico Mesoamericano. World Resources Institute, Washington DC.

Ciarniello, L. M., Boyce, M. S., Seip, D. R., \& Heard, D. C. (2007). Grizzly bear habitat selection is scale dependent. Ecological Applications, 17(5), 1424-1440. doi: 10.1890/06-1100.1

Clark, M., \& Tilman, D. (2017). Comparative analysis of environmental impacts of agricultural production systems, agricultural input efficiency, and food choice. Environmental Research Letters, 12(6), 064016. doi: 10.1088/1748-9326/aa6cd5

Clerici, N., Salazar, C., Pardo-Díaz, C., Jiggins, C. R., \& Linares, M. (2018). Peace in Colombia is a critical moment for Neotropical connectivity and conservation: Save the northern Andes-Amazon biodiversity bridge. Conservation Letters, 12(1), e12594. doi: $\underline{10.1111 / \text { conl.12594 }}$

Convention on Biological Diversity [CDB] (2010). Decision $\mathrm{UNEP} / \mathrm{CBD} / \mathrm{COP} / \mathrm{DEC} / \mathrm{X} / 2$. Adopted by the Conference of the Parties to the Convention on Biological Diversity at its Tenth Meeting. Recuperado de https://www.cbd.int/decision/cop/?id=12268

Corlett, R.T. (2015). The anthropocene concept in ecology and conservation. Trends in Ecology \& Evolution, 30(1), 36-41. doi: 10.1016/i.tree.2014.10.007.

Correa-Ayram, C. A., Mendoza, M. E., Etter, A., \& Pérez-Salicrup, D. R. (2017). Anthropogenic impact on habitat connectivity: a 
multidimensional human footprint index evaluated in a highly biodiverse landscape of Mexico. Ecological Indicators, 72, 895-909. doi: 10.1016/j.ecolind.2016.09.007

Correa-Ayram, C., Mendoza, M. E., Etter, A., \& Pérez-Salicrup, D. R. (2016). Habitat connectivity in biodiversity conservation: a review of recent studies and applications. Progress in Physical Geography: Earth and Environment, 40(1), 7-37. doi: $\underline{10.1177 / 0309133315598713}$

Correa-Ayram, C., Mendoza, M. E., Pérez-Salicrup, D. R., \& López Granados, E. (2014) Identifying potential conservation areas in the Cuitzeo lake basin, Mexico by multitemporal analysis of landscape connectivity. Journal for Nature Conservation, 22(5), 424435. doi: 10.1016/j.jnc.2014.03.010

Correa-Ayram, C.A., Etter, A., Díaz-Timoté, J., Rodríguez-Buriticá, S., Ramírez, W., \& Corzo, G. (2020). Spatiotemporal evaluation of the human footprint in Colombia: Four decades of anthropic impact in highly biodiverse ecosystems. Ecological Indicators, 117, 106630. doi: 10.1016/i.ecolind.2020.106630

Coulon, A., Cosson, J. F., Angibault, J. M., Cargnelutti, B., Galan, M., Morellet, N., Petit, E., Aulagnier, S., \& Hewison, A. J. M. (2004). Landscape connectivity influences gene flow in a roe deer population inhabiting a fragmented landscape: an individualbased approach. Molecular Ecology, 13(9), 2841-2850. doi: 10.1111/j.1365-294X.2004.02253.x

Cushman, S.A. (2006). Effects of habitat loss and fragmentation on amphibians: A review and prospectus. Biology and Conservation. 128(2), 231-240. doi: 10.1016/j.biocon.2005.09.031

DeFries, R. S., Rudel, T., Uriarte, M., \& Hansen, M. (2010). Deforestation driven by urban population growth and agricultural trade in the twenty-first century. Nature Geoscience, 3, 178-181. doi: $\underline{10.1038 / \mathrm{NGEO} 756}$

Diniz, M. F., Machado, R. B., Bispo, A. A., \& Brito, D. (2017). Identifying key sites for connecting jaguar populations in the Brazilian Atlantic Forest. Animal Conservation, 21(3), 201-210. doi: $\underline{10.1111 / \text { acv.12367 }}$

Elston, D. A., \& Bucland, S. T. (1993). Statistical modelling of regional GIS data and overview. Ecological Modelling, 67(1), 81-102.

Etterson, J. R., \& Mazer, S. J. (2016). How climate change affects plants' sex lives. Science, 353(6294), 32-33. doi: $\underline{10.1126 / \text { science.aag1624 }}$

Eva, H. D., Achard, F., Beuchle, R., De Miranda, E., Carboni, S., Seliger, R., Vollmar, Michael., Holler, W. A., Oshiro, O. T., BarrenaArroyo, V., \& Gallego, J. (2012). Forest Cover Changes in Tropical South and Central America from 1990 to 2005 and
Related Carbon Emissions and Removals. Remote Sensing, 4(5), 1369-1391. doi: 10.3390/rs4051369

Fazey, I., Fischer, J. \& Lindenmayer, D. B. (2005). What do conservation biologists publish? Biological Conservation, 124(1), 63-73. doi: $\underline{10.1016 / \text { j.biocon.2005.01.013 }}$

Foley, J.A., DeFries, R., Asner, G. P., Barford, C., Bonan, G., Carpenter, S. R., Stuart, F., Chapin., Coe, M. T., Daily, G. C., Gibbs, H. K., Helkowski, J. H., Holloway, T., Howard, E. A., Kucharick, C. J., Monfreda, C., Patz, J. A., Prentice, I. C., Ramankutty, N., \& Synder, P.K. (2005). Global consequences of land use. Science, 309(5734), 570-74. doi: 10.1126/science.1111772

Forman, R. T. T. (1994). Land mosaics, the ecology of landscapes and regions. University Press, Cambridge.

FRA [Evaluación de los Recursos Forestales Mundiales]. (2015). Organización de las Naciones Unidas para la Alimentación y la Agricultura. Compendio de datos. Roma. pp 253.

Furtado, C. (2007). A economia latino-americana: formacao histórica e problemas contemporaneous. Companhia das Letras, Sao Paulo.

Garcia, R. A., Cabeza, M., Rabhek, C., \& Araujo, M. B. (2014). Multiple dimensions of climate change and their implications for biodiversity. Science, 344(6183), 6183; 1247579. doi: $\underline{10.1126 / \text { science. } 1247579 .}$.

Geist, H. J., \& Lambin, E. F. (2001). What drives tropical deforestation? A meta-analysis of proximate and underlying causes of deforestation based on subnational case study evidence. LUCC Report Series, 4, $116 . \quad \underline{\text { https://www.pik- }}$ potsdam.de/members/cramer/teaching/0607/

Geist, H. J., \& Lambin, E. F. (2002). Proximate causes and underlying driving forces of tropical deforestation. Bioscience 52(2), 143-150. doi: $10.1641 / 0006-35682$

Gilbert-Norton, L., Wilson, R., Stevens, J. R., \& Beard, K. H. (2010). A meta-analytic review of corridor effectiveness. Conservation Biology, 24(3), 60-668. doi: 10.1111/j.1523-1739.2010.01450.x

Groves, C. R., Game, E. T., Anderson, M. G., Cross, M., Enquist, C., Ferdaña, Z., Girvetz, E., Gondor, A., Hall, K. R., Higgins, J., Marshall, R., Popper, K., Schill, S., \& Shafer, S. L. (2012). Incorporating climate change into systematic conservation planning. Biodiversity and Conservation, 21, 1651-1671. doi: 10.1007/s10531-012-0269-3.

Haila, Y. (2002). A conceptual genealogy of fragmentation research: from island biogeography to landscape ecology. Ecological Applications, 12(2), 321-334. doi: 10.1890/1051-0761 
Hannah, L., Midgley, G. F., \& Millar, D. (2002). Climate changeintegrated conservation strategies. Global Ecology and Biogeography, 11(6), 485-495.

Hansen, M. C., Potapov, P. V., Moore, R., Hancher, M., Turubanova, S. A., Tyukavina, A., Thau, D., Stehman, S. V., Goetz, J., Loveland, T. R., Kommareddy, A., Egorov, A., Chini, L., Justice, C. O., \& Townshend, J. R.G. (2013). High-resolution global maps of 21stcentury forest cover change. Science, 342(6160), 850-853. doi: $\underline{10.1126 / \text { science. } 1244693}$

Hardt, E., Borgomeo, E., dos Santos, R. F., Pinto, G. L. F., Metzger, J. P., \& Sparovek, G. (2015). Does certification improve biodiversity conservation in Brazilian coffee farms. Forest Ecology and Management, 357, 181-194. doi: 10.1016/j.foreco.2015.08.021

Harrison, P. A., Vandewalle, M., Sykes, M. T., Berry, P. M., Bugter, R., de Bello, F., Feld, C. K., Grandin, U., Harrington, R., Haslett, J. R., Jongman, R. H. G., Luck, G.W., Martins da Silva, P., Moora, M., Settele, J., Sousa, J. P., \& Zobel, M. (2010). Identifying and prioritising services in European terrestrial and freshwater ecosystems. Biodiversity and Conservation, 19(10), 2791-2821. doi: $\underline{10.1007 / \mathrm{s} 10531-010-9789-\mathrm{x}}$

Hatfield, J. H., Orme, C. D., \& Banks-Leite, C. (2018). Using functional connectivity to predict potential meta-population sizes in the Brazilian Atlantic Forest. Perspectives in Ecology and Conservation, 16(4), 215-220. doi: 10.1016/i.pecon.2018.10.004

Hill, J. K., Griffiths, H. M., \& Thomas, C. D. (2011). Climate change and evolutionary adaptations at species' range margins. Annual Review of Entomology, 56, 143-159. doi: 10.1146/annurev-ento-120709$\underline{144746}$

Hobbs, R. J., \& Yates, C. J. (2003). Impacts of ecosystem fragmentation on plant populations: generalizing the idiosyncratic. Australian Journal of Botany, 51, 471-488. doi: 10.1071/BT03037

Hoffmann, A. A., \& Sgro, C. M. (2011). Climate change and evolutionary adaptation. Nature, 470, 479-485. doi: 10.1038/nature09670

Höglund, J. (2009). Evolutionary conservation genetics. Oxford University Press: Oxford.

Horta, M. B., Bhakti, T., Cordeiro, P.F., Carvalho-Ribeiro, A. M., Fernandes, G. W., \& Goulart, F. F. (2018). Functional connectivity in urban landscapes promoted by Ramphastos toco (Toco Toucan) and its implications for policy formulation. Urban Ecosystems, 21, 1097-1111. doi: 10.1007/s11252-018-0789-Z

Imbach, P. A., Locatelli, B., Molina, L. G., Clais, P., \& Leadley, P. W. (2013). Climate change and plant dispersal along corridors in fragmented landscapes of Mesoamerica. Ecology and Evolution, 3(9), 2917-2932. doi: 10.1002/ece3.672
Intergovernmental Panel on Climate Change [IPCC]. (2007). Climate change 2007: synthesis report. Contribution of working groups I, II and III to the fourth assessment report of the intergovernmental panel on climate change. Geneva, Switzerland: IPCC.

Junhu, S., Achyut, A., Ibrahim, M., Hegab, U., Babu, S., Sean, C.P., Coogan, S., Sathyakumar, M., Dalannast, Z., Dou, Y., Suo, X., Dabu, H., Fu, L., \& Wu, W. (2018). Decreasing brown bear (Ursus arctos) habitat due to climate change in Central Asia and the Asian Highlands. Ecology and Evolution. 8(23), 11887-11899. doi: $10.1002 /$ ece 3.4645

Kacowicz, A. M. (2008). Latin America and the world: Globalization, regionalization, and fragmentation. Nueva Sociedad, 214, 1-11.

Kim, D. H., Sexton, J. O., \& Townshend, J. R. (2015). Accelerated deforestation in the humid tropics from the 1990s to the 2000s. Geophysical Research Letters, 42(9), 495-3501. ㅇi: $\underline{10.1002 / 2014 G L 062777}$

Krosby, M., Tewksbury, J., Haddad, N. M., \& Hoekstra, J. (2010). Ecological connectivity for a changing climate. Conservation Biology, 24(6), 1686-1689. doi: 10.1111/j.1523-1739.2010.01585.x

Laestadius, L., Maginnis, S., Minnemeyer, S., Potapov, P., Saint-Laurent, C., \& Sizer, N. (2011). Mapping opportunities for forest landscape restoration. Unasylva, 62, 238.

Lambin, E.F. 1994. Modelling deforestation process a review. European Commission, Institute for Remote Sensing Applications. European Space Agency, 113 pp.

Lambin, E. F. (1997). Modeling and monitoring land-cover change processes in tropical regions. Progress in Physical Geography, 21(3), 375-393. doi: $10.1177 / 030913339702100303$

Lambin, E. F., Geist, H. J., \& Lepers, E. (2003). Dynamics of land-use and land-cover change in tropical regions. Annual Review of Environment and Resources, 28, 205-241. doi: 10.1146/annurev.energy.28.050302.105459

Lambin, E. F., Turner, B. L., Geist, H. J., Agbola, S. B., Angelsen. A., Bruce, J. W., Coomes, O. T., Dirzo, R., Fischerh, G., Folke, C., George, P. S., Homewood, K., Imbernon, J., Leemans, R., Li, X., Moran, E. F., Mortimore, M., Ramakrishnan, P. S., Richards, J. F., Skanes, H., Steffen, W., Stone, G. D., Svedin, U., Veldkamp, T. A., Vogel, C., \& Xu, J. (2001). The causes of land-use and landcover change: moving beyond the myths. Global Environmental Change, 11(4), 261-269. doi: 10.1016/S0959-3780(01)00007-3

Laurance, W. F., Camargo, J. L., Luizão, R. C., Laurance, S. G., Pimm, S. L., Bruna, E. M., Stouffer, P. C., Williamson, G. B., Ben, J., Tez-Malvido, H. L., Vasconcelos, K. S., Van-Houtan, C. E., Zartman, S. A., Boyle, R. K., Didham, A., Andrade., \& Lovejoy, T.E. (2011). The fate of Amazonian forest fragments: a 32-year 
investigation. Biological Conservation, 144(1), 56-67. doi: 10.1016/j.biocon.2010.09.021

Lavelle, P., Rodríguez, N., Arguello, O., Berna, J., Botero, C., Chaparro, P., Gómez, Y., Gutiérrez, A., Hurtado, M., Loaiza, S., Pullido, S. X., Rodríguez, E., Sanabria, C., Velásquez, E., \& Fonte, S.J. (2014). Soil ecosystem services and land use in the rapidly changing Orinoco River Basin of Colombia. Agriculture, Ecosystems and Environment, 185, 106-117. doi: 10.1016/j.agee.2013.12.020

Leidner, A.K., \& Haddad, N.M. (2011). Combining measures of dispersal to identify conservation strategies in fragmented landscapes. Conservation Biology, 25(5), 1022-1031. doi: 10.1111/j.1523-1739.2011.01720.x.

Leija, E. G., Reyes-Hernández, H., Reyes-Pérez, O., Flores-Flores, J. L., \& Sahagún, F. J. S. (2016). Cambios en la cubierta vegetal, usos de la tierra y escenarios futuros en la región costera del estado de Oaxaca, México. Madera y Bosques 22(1), 125-140. doi: 10.21829/myb.2016.221481

Leija, E.G., \& Pavón, N. P. (2017). The northernmost tropical rain forest of the Americas: endangered by agriculture expansion. Tropical Ecology, 58(3), 641-652.

Leija, E.G., Valenzuela-Ceballos, S. I., Valencia-Castro, M., JiménezGonzález, G., Castañeda-Gaytán, G., Reyes-Hernández, H., Mendoza, M. E. (2020). Análisis de cambio en la cobertura vegetal y uso del suelo en la región centro-norte de México. El caso de la cuenca baja del río Nazas. Ecosistemas, 29(1), 1826. doi: $\underline{10.7818 / \mathrm{ECOS} .1826}$

Lindenmayer, D., \& Fischer, J. (2006). Fragmentación del bábitat y cambio de paisaje: una sintesis ecológica y de conservación. Washington, DC, EE. UU: Island Press.

Lindenmayer, D., S. Cunningham, \& Young, A. (2012). Perspectives on land use intensification and biodiversity conservation. En D. Lindenmayer, S. Cunningham, \& A. Young (Eds.), Land use intensification: effects on agriculture, biodiversity and ecological processes (pp. 123-134), Boca Raton, Florida, USA: CRC Press.

Liu, F., McShea, W., Garshelis, D., Zhu, X., Wang, D., Gong, J., \& Chen, Y. (2009). Spatial distribution as a measure of conservation needs: an example with Asiatic black bears in south-western China. Diversity and Distributions, 15(4), 649- 659. doi: 10.1111/j.1472$\underline{4642.2009 .00571 . x}$

Loyola, R. D., Lemes, P., Faleiro, F. V., Trindade-Filho, J., \& Machado, R. B. (2012). Severe loss of suitable climatic conditions for marsupial species in Brazil: Challenges and opportunities for conservation. Plos One, 7(9), e46257 doi: 10.1371/journal.pone.0046257
Luque, S., Saura, S., \& Fortin, M. J. (2012). Landscape connectivity analysis for conservation: Insights from combining new methods with ecological and genetic data. Landscape Ecology, 27(2), 153-157. doi: $10.1007 / \mathrm{s} 10980-011-9700-5$

Martínez-Ramos, M., Pingarroni, A., Rodríguez-Velázquez, J., ToledoChelala, L., Zermeño-Hernández, I. \& Bongers, F. (2016). Natural forest regeneration and ecological restoration in humanmodified tropical landscapes. Biotropica, 48(6), 745-757. doi: $\underline{10.1111 / \text { btp. } 12382}$

Martín-López, B., Iniesta-Arandia, I., García-Llorente, M., Palomo, I., Casado-Arzuaga, I., Amo, D. G., Gómez-Baggethun, E., OterosRozas, E., Palacios-Agundez, I., Willaarts, B., González, J. A., Santos-Martín, F., Onaindia, M., López-Santiago, C., \& Montes, C. (2012a). Uncovering ecosystem service bundles through social preferences. PloS One, 7(6), e38970. doi: 10.1371/journal.pone. 0038970

Martins, J. H., Camanho, A. S. \& Gaspar, M. B. (2012). A review of the application of driving forces-pressure-state-impact-response framework to fisheries management. Ocean Coastal Manage, 69, 273-281. doi: 10.1016/j.ocecoaman.2012.07.029

Maskell, L. C., Crowe, A., Dunbar, M. J., Emmett, B., Henrys, P., Keith, A. M., Norton, L. R., Scholefield, P., Clark, D. B., Simpson, I. C., \& Smart, S. M. (2013). Exploring the ecological constraints to multiple ecosystem service delivery and biodiversity. Journal of Applied Ecology, 50(3), 561-571. doi: 10.1111/1365-2664.12085

Mathieu, J., Grimaldi, M., Jouquet, P., Rouland, C., Lavelle, P., Desjardins, T., \& Rossi, J. P. (2009). Spatial patterns of grasses influence soil macrofauna biodiversity in Amazonian pastures. Soil Biology and Biochemistry, 41(3), 586-593. doi: $\underline{10.1016 / \text { i.soilbio. } 2008.12 .020}$

McRae, B. H., Hall, S. A., \& Beier, P. (2012). Where to restore ecological connectivity? Detecting barriers and quantifying restoration benefits. PloS One, 7(12), e52604 doi: 10.1371/journal.pone.0052604

Mech, S. G., \& Hallet, J. G. (2001). Evaluating the effectiveness of corridors: a genetic approach. Conservation Biology, 15(2), 467-474.

Mendoza, M. E., Lopéz, G. E., Geneletti, D., Pérez-Salicrup, D. R., \& Salinas, V. (2011). Analysing land cover and land use change processes at watershed level: A multitemporal study in the Lake Cuitzeo Watershed, Mexico (1975-2003). Applied Geograpghy. 31(1), 237-250. doi: 10.1016/j.apgeog.2010.05.010

Millenium Ecosystem Assessment [MEA]. (2005). Ecosystem and human well-being: A framework for assessment. 4 volumes. Island Press. Washington. D.C, EE. UU. 
Mitchell, M. E., Bennett, E. \& Gonzalez, A. (2013). Linking landscape connectivity and ecosystem service provision: current knowledge and research gaps. Ecosystems, 16(5), 894- 908. doi: $\underline{10.1007 / \mathrm{s} 10021-013-9647-2}$

Mony, C., Abadie, J., Gil-Tena, A., Burel, F., \& Ernoult, A. (2018). Effects of connectivity on animal-dispersed forest plant communities in agriculture-dominated landscapes. Journal of Vegetation Science, 29(2), 167-178. doi: 10.1111/jvs.12606

Mora, C., Frazier, A. G., Longman, R. J., Dacks, R. S., Walton, M. M., Tong, E. J., Sanchez, J. J., Kaiser, L. R., Stender, Y. O., Anderson, J. M., Christine, M., Fernandez-Silva, A. I., Louise, M., Giuseffi., \& T. W. Giambelluca (2013). The projected timing of climate departure from recent variability. Nature, 502, 183-187. doi: $\underline{10.1038 / \text { nature } 12540}$

Moraes, A. M., Grativol, A. D., De Vleeschouwer, K. M., Ruiz-Miranda, C. R., Raboy, B. E., Oliveira, L.C., Dietz, J. M., \& Galbusera, P. H. A. (2018). Population genetic structure of an endangered endemic primate (Leontopithecus chrysomelas) in a highly fragmented Atlantic coastal rain forest. Folia Primatologica, 89, 365381. doi: $10.1159 / 000492176$

Morera, C., Pintó, J., \& Romero, M. (2007). Paisaje, procesos de fragmentación y redes ecológicas: aproximación conceptual. En O. Chassot \& C. Morera. (Eds.), Corredores biológicos: acercamiento conceptualy experiencia en América (pp. 11-47). San José, Costa Rica: Imprenta Nacional.

Múgica, M., De Lucio, J. V., Martínez, C., Sastre, P., Atauri, J.A., \& Montes, C. (2002). Integración territorial de espacios naturales protegidos y conectividad ecológica en paisajes mediterráneos. Servicios Ambientales Consejería de Medio Ambiente Junta de Andalucía, España.

Newbold, T., Hudson, L. N., Hill, S. L., Contu, S., Lysenko, I., Senior, R. A., Börger, L., Bennett, D. J., Choimes, A., Collen, B., Day, J., De Palma, A., Díaz, S., Echeverria-Londoño, S., Edgar, M. J., Fieldman, A., Garon, M., Harrison, M. L., Alhusseini, T., Ingram, D. J., Itescu, Y., Kattge, J., Kemp, V., Krikpatrick, L., Kleyer, M., Correia, D. L., Martin, C. D., Meiri, S., Novosolov, M., Pan, Y., Phillips, H. R., Purves, D. W., Robinson, A., Simpson, J., Tuck, S. L., Weiher, E., White, H. J., Ewers, R. M., Mace, G. M., Scharlemann, J. P., \& Purvis, A. (2015). Global effects of land use on local terrestrial biodiversity. Nature, 520, 45-50. doi: $\underline{10.1038 / \text { nature14324 }}$

Oliveira, U., Soares-Filho, B., Machado-Leitao, R. F., \& Rodrigues, H. O. (2019). BioDinamica: a toolkit for analyses of biodiversity and biogeography on the Dinamica-EGO modelling platform. PeerJ, 7, e7213 doi: $10.7717 /$ peer.j.7213

Opdam, P. F. M., Verboom, J., \& Pouwels, R. (2003). Landscape cohesion: an index for the conservation potential of landscapes for biodiversity. Landscape Ecology, 18, 113-126. doi: 10.1023/A:1024429715253

Parks, S. A., McKelvey, K. S., \& Schwartz, M. K. (2013). Effects of weighting schemes on the identification of wildlife corridors generated with least-cost methods. Conservation Biology, 27(1), 145154. doi: 10.1111/j.1523-1739.2012.01929.x

Pettorelli, N., Safi, K., \& Turner, W. (2014). Satellite remote sensing, biodiversity research and conservation of the future. Philosophical transactions of the Royal Society. Biological Sciences, 369(1643), 20130190. doi: 10.1098/rstb.2013.0190.

Piquer-Rodríguez, M., Kuemmerle, T., \& Alcaraz-Segura, D. (2012). Future land use effects on the connectivity of protected area networks in southeastern Spain. Journal for Nature Conservation, 20(6), 326-336. doi: 10.1016/j.jnc.2012.07.001

Pound, M. J., \& Salzmann, U. (2017). Heterogeneity in global vegetation and terrestrial climate change during the late Eocene to early Oligocene transition. Scientific Reports, 7, 43386. doi: $\underline{10.1038 / \text { srep } 43386}$

Quesada, M., Sanchez-Azofeifa, G. A., Alvarez-Añorve M., Stoner, K. E., Avila-Cabadilla, L., Calvo-Alvarado, J., Castillo A., EspíritoSanto, M. M., Fagundes, M., Fernandes, G.W., Gamonb, J., Lopezaraiza-Mikel, M., Lawrence, D., Cerdeira-Morellato, L.D., Powers, J. S., Neves, F. S., Rosas-Guerrero, V., Sayago, R., \& Sanchez-Montoya, G. 2009. Succession and management of tropical dry forests in the Americas: Review and new perspectives. Forest Ecology and Management, 258(6), 1014-1024. doi: $\underline{10.1016 / j . f o r e c o .2009 .06 .023}$

Santos, M. (2010). Por una outra globalização: do pensamento único á consciéncia universal. Rio de Janeiro: Record.

Sarukhán, J. (2007). Editorial. Boletín de la Sociedad Botánica de México, 80 (Suplemento), 7-10.

Saura, S., Bastin, L., Battistella, L., Mandrici, A. \& Dubois, G. (2017). Protected areas in the world's ecoregions: how well connected are they? Ecological Indicators, 76, 144-158. doi: $\underline{10.1016 / j . e c o l i n d .2016 .12 .047}$

Saura, S., Bertzky, B., Bastin, L., Battistella, L., Mandrici, A., \& Dubois, G. (2019). Global trends in protected area connectivity from 2010 to 2018. Biological Conservation, 238, 108183. doi: $\underline{10.1016 / \text { j.biocon.2019.07.028 }}$

Sayer, J., Sunderland, T., Ghazoul, J., Pfund, J. L., Sheil, D., Meijaard, E., \& Buck, L. E. (2013). Ten principles for a landscape approach to reconciling agriculture, conservation, and other competing land uses. PNAS, 110(21), 8349-8356. doi: 10.1073/pnas.1210595110

Smith, A.C., Fahrig, L., \& Francis, C. M. (2011). Landscape size affects the relative importance of habitat amount, habitat fragmentation, 
and matrix quality on forest birds. Ecography, 34(1), 103-113. doi: 10.1111/j.1600-0587.2010.06201.x

Sodhi, N. S., Koh, L.P., Brook, B. W., \& Ng, P. K. (2004). Southeast Asian biodiversity: an impending disaster. Trends in Ecology \& Evolution, 19(12), 654-660. doi: 10.1016/i.tree.2004.09.006

Sohoulande, D. D. C. (2017). Spectrum of climate change and streamflow alteration at a watershed scale. Environmental Earth Sciences, 76, 653. doi: 10.1007/s12665-017-7006-x

Stoufier, P. C. \& Bierregaard, R. O. (1995). Effects of forest fragmentation on understory hummingbirds in Amazonian Brazil. Conservation Biology, 9(5), 1085-1094.

Stuart, S. N., Chanson, J. S., Cox, N. A., Young, B. E., Rodrigues, A. S. L., Fischman, D. L. \& Waller, R. W. (2004). Status and trends of amphibian declines and extinctions worldwide. Science, 306(5702), 1783-1786. doi: 10.1126/science. 1103538

Suding, K. N., Gross, K. L., \& Houseman, G. R. (2004). Alternative states and positive feedbacks in restoration ecology. Trends in Ecolology \& Evolution, 19(1), 46-53. doi: 10.1016/j.tree.2003.10.005

Suding, K., Higgs, E., Palmer, M., Callicott, J. B., Anderson, C. B., Baker, M., \& Schwartz, K. Z. S. (2015). Committing to ecological restoration. Science, 348(5235), 638- 640. doi: $\underline{10.1126 / \text { science.aaa4216 }}$

Summers, D. M., Bryan, B. A., Crossman, N. D., \& Meyer, W. S. (2012). Species vulnerability to climate change: impacts on spatial conservation priorities and species representation. Global Change. Biology, 18(7), 2335-2348. doi: 10.1111/j.1365-2486.2012.02700.x

Tambosi, L. R., \& Metzger, J. P. (2013). A framework for setting local restoration priorities based on landscape context. Natureza \& Conservação, 11(2), 152-157. doi: 10.4322/natcon.2013.024

Taylor, P. D., Fahrig, L., Henein, K., \& Merriam, G. (1993). Connectivity is a vital element of landscape structure. Oikos, 68(3), 571-573. doi: $10.2307 / 3544927$

Taylor, P.D., Fahrig, L., With, K. A. (2006). Landscape connectivity: A return to the basics. En Crooks, K. R. \& M. Sanjayan, (Eds.), Connectivity conservation (29-43). Cambridge, UK: Cambridge University Press.

Tischendorf, L., \& Fahrig, L. (2000). On the usage and measurement of landscape connectivity. Oikos, 90(1), 7-19. doi: 10.1034/i.1600$\underline{0706.2000 .900102 . x}$

Townshend, J. R., Masek, J. G., Huang, C., Vermote, E. F., Gao, F., Channan, S., Sexton, J.O., Feng, M., Narasimhan, R., Kim, D.,
Song, K., Song, D., Song, X. P., Noojipaday, P., Tan, B., \& Hansen, M. C. (2012). Global characterization and monitoring of forest cover using Landsat data: opportunities and challenges. International Journal of Digital Earth, 5(5), 373-397. doi: $\underline{10.1080 / 17538947.2012 .713190}$

Turner, M., \& Gardner, R. (1990). Quantitative methods in landscape ecology. Nueva York: Springer-Verlag.

Varga-Lopes, E., Mendonça, B. L., Dos Santos, J., Augusto, Marcelo., López-Iborra, G., \& Dos Anjos, M. L. (2016). Effects of connectivity on the forest bird communities of adjacent fragmented landscapes. Ardeola, 63(2), 279-293. doi: $\underline{10.13157 / \text { arla.63.2.2016.ra4 }}$

Vos, C., Berry, P., Opdam, P., Baveco, H., Nijhof, B., O'hanley, J., Bell, C., \& Kuipers, H. (2008). Adapting landscapes to climate change: examples of climate-proof ecosystem networks and priority adaptation zones. Journal of Applied Ecology, 45(6), 1722-1731. doi: $\underline{10.1111 / j .1365-2664.2008 .01569 . x}$

Wan, H., Rasch, P. J., Zhang, K., Qian, Y., Yan, H., \& Zhao, C. (2014). Short ensembles: An efficient method for discerning climaterelevant sensitivities in atmospheric general circulation models. Geoscientifc Model Development, 7, 1961-1977. doi: 10.5194/gmd-71961-2014.

Wikelski, M., \& Tertitski, G. (2016). Living sentinels for climate change effects. Science, 352(6287), 775-776. doi: 10.1126/science.aaf6544

Wright, J. S. (2005). Tropical forests in a changing Environment. Ecology and Evolution, 20(10), 553-560. doi: 10.1016/j.tree.2005.07.009

Wu, X., Lu, Y., Zhou, S., Chen, L., \& Xu, B. (2016). Impact of climate change on human infectious diseases: Empirical evidence and human adaptation. Environment International, 86, 14-23. doi: $\underline{10.1016 / \text { i.envint.2015.09.007 }}$

Zacarias, D., Loyola, R. (2018). Distribution modelling and multi-scale landscape connectivity highlight important areas for the conservation of savannah elephants. Biological Conservation, 224, 1 8. doi: 10.1016/j.biocon.2018.05.014

Zeller, K., McGarigal, K., \& Whiteley, A. (2012). Estimating landscape resistance to movement: a review. Landscape Ecology, 27, 777-797. doi: 10.1007/s10980-012-9737-0

Zimbres, B., Peres, C.A., \& Machado, R. B. (2016). Terrestrial mammal responses to habitat structure and quality of remnant riparian forests in an Amazonian cattle-ranching landscape. Biological Conservation, 206, 283-292. doi: 10.1016/j.biocon.2016.11.033 
Manuscrito recibido el 27 de agosto de 2019

Aceptado el 19 de mayo de 2020

Publicado el 19 de marzo de 2021

Este documento se debe citar como:

Leija, E. G. \& Mendoza, M. E. (2O21). Estudios de conectividad del paisaje en América Latina: retos de investigación. Madera y Bosques, 27(1), e2712032. doi: 10.21829/myb.2021.2712032
Madera y Bosques por Instituto de Ecología, A.C. se distribuye bajo una Licencia Creative Commons Atribución-NoComercialCompartirlgual 4.0 Internacional. 